\title{
Solution of the NLO BFKL Equation and a Strategy for Solving the All-Order BFKL Equation
}

\author{
Giovanni A. Chirilli,* Yuri V. Kovchegov ${ }^{\dagger}$ \\ Department of Physics, The Ohio State University, Columbus, OH 43210, USA
}

\begin{abstract}
We derive the solution of the NLO BFKL equation by constructing its eigenfunctions perturbatively, using an expansion around the LO BFKL (conformal) eigenfunctions. This method can be used to construct a solution of the BFKL equation with the kernel calculated to an arbitrary order in the coupling constant.
\end{abstract}

PACS numbers: 12.38.-t, 12.38.Bx, 12.38.Cy

\section{INTRODUCTION}

At very high energy the dynamics of hadronic and nuclear scattering processes is dominated by non-linear effects associated with parton saturation (see [1 [5] for the reviews of the subject). Scattering amplitudes of these processes can be factorized in rapidity-space using the operator product expansion in terms of Wilson-lines, infinite gauge links ordered along the straight line collinear to the particle's velocity close to the light-cone [6]. The evolution of Wilson-lines with respect to rapidity is given by the non-linear Balitsky-Kovchegov (BK) $7-10$ ] and Jalilian-MarianIancu-McLerran-Weigert-Leonidov-Kovner (JIMWLK) [1] 14 evolution equations. The pre-asymptotic behavior of the scattering amplitude, when the parton density is not yet high enough for saturation effects to become important, is well-described by the linear Balitsky-Fadin-Kuraev-Lipatov (BFKL) 15-17] evolution equation.

Over the last decade the physics of saturation/Color Glass Condensate (CGC) achieved a number of phenomenological successes, in both predicting and describing the experimental data (see e.g. [18, 19]). Current phenomenological applications mainly employ the BK evolution in the leading logarithmic approximation (LLA), which resums powers of $\alpha_{s} \ln s$, with the running coupling corrections included in the evolution kernel 20 23]. (Here $\alpha_{s}$ is the strong coupling and $s$ is the center-of-mass energy.) Further progress in terms of precision of the CGC predictions and in understanding the accuracy of the CGC fits can be achieved by studying other higher-order corrections to the BFKL/BK/JIMWLK evolution equations. It is, therefore, very important to better understand the structure of high-order corrections to the small- $x$ evolution equations.

At the moment, the full next-to-leading-order (NLO) kernel is known for the BFKL [24, 25] and BK [26] equations, though phenomenological implementation of the latter is presently lacking. The calculation of the NLO BFKL kernel some fifteen years ago 24, 25] spurred a vigorous activity trying to understand the effects of NLO corrections on the solution of the leading-order (LO, or LLA) BFKL equation.

The solution of the LO BFKL evolution [15 17] describes a scattering amplitude that grows proportionally to a positive power of the center of mass energy of the hadronic or nuclear scattering process: at this order the kernel of the evolution equation respects the conformal symmetry of the $S L(2, C)$ Möbius group and the eigenfunctions are power-like functions of transverse distance in coordinate space (or, in momentum space, powers of transverse momenta), while the eigenvalue of the kernel is related to the Pomeron intercept. At the NLO there appears also a contribution to the evolution kernel due to the running of the QCD coupling constant and the conformal property of the LO BFKL is lost. Consequently, the LO BFKL kernel's conformal eigenfunctions are not eigenfunctions of the NLO BFKL kernel: at this order the power-law growth of the amplitudes with energy also seems to be lost because of the non-Regge terms appearing due to the running coupling effects 27 30]. It was also found that the NLO BFKL corrections generate a large negative contribution to the eigenvalue/Pomeron intercept 24, 31 33], though resummations of collinear divergences to all higher orders in the kernel offer a promising way to tame this large correction 34 36].

Despite a number of efforts, it appears that an exact analytical solution of the NLO BFKL equation, or a general all-order BFKL equation in QCD is still lacking (see e.g. 37|). This is in stark contrast to the DokshitzerGribov-Lipatov-Altarelli-Parisi (DGLAP) evolution equation [38 40], which is a renormalization group equation in the virtuality $Q^{2}$ : the eigenfunctions of that evolution equation are simple powers of Bjorken- $x$ variable for the kernel calculated to any order in the coupling constant. The general form of the solution for DGLAP equation is well-known,

\footnotetext{
* chirilli.1@asc.ohio-state.edu

$\dagger$ kovchegov.1@asc.ohio-state.edu
} 
with the higher-order corrections in the powers of the coupling constant entering into the anomalous dimension of the operator at hand [40 44].

The goal of this work is to devise a systematic way of solving the BFKL evolution equation order-by-order in the perturbation theory. We consider scattering in the pre-asymptotic regime, where the non-linear saturation corrections due to BK/JIMWLK evolution are not yet important, and the linear BFKL equation gives a good description of the scattering amplitude.

Note that in the $\mathcal{N}=4$ super-Yang-Mills (SYM) theory, which is a conformal field theory without the running of the coupling, the form of the solution of the all-order BFKL equation is known [45 48]: conformal symmetry fixes the eigenfunctions of the BFKL kernel in $\mathcal{N}=4$ SYM theory to be the eigenfunctions of the Casimir operators of the Möbius group, $E^{n, \nu}$ [49], and the perturbative expansion is confined to the eigenvalues/the intercept.

In QCD the situation is not so straightforward, since, as we have already mentioned, running coupling corrections destroy the conformal symmetry of the BFKL kernel, such that the eigenfunctions of NLO (and higher-order) BFKL equation are not known. Below we will construct the eigenfunctions of the NLO BFKL by a perturbative expansion in powers of $\alpha_{s}$ around the conformal eigenfunctions of the LO BFKL kernel. Naturally the corrections we find are proportional to the QCD beta-function. Knowing the eigenfunctions and eigenvalues allows us to find the exact solution of the NLO BFKL equation, given by Eq. (50) below. (For simplicity we are working in the limit where the amplitudes do not depend on the azimuthal angles of the transverse momenta, which corresponds to the dominant high-energy asymptotics.) Our procedure can be applied to any higher-order BFKL kernel, once it is calculated. We thus see that the general solution of the BFKL equation in QCD using our technique contains a perturbative expansion both in the eigenfunctions and in the eigenvalues, which give the BFKL Pomeron intercept. This is our proposal for organizing the perturbation series for the solution of the BFKL equation.

The paper is structured as follows: in Sec. II we formulate the problem of finding the all-order BFKL Green function. The NLO eigenfunctions and eigenvalues are found in Sec. III and are given in Eqs. (47) and (49) correspondingly. We see that the eigenvalues we found are the same as that commonly used in the literature (see e.g. [31]). The solution for the NLO BFKL equation is given in Eq. (50). The general form of the solution for the all-order BFKL equation is given in Sec. IV by Eq. (52). The properties of our solution of the NLO BFKL equation are studied in Sec. $\mathrm{V}$. in which we demonstrate the renormalization scale independence of the obtained solution (within the perturbative precision). We also rewrite the obtained NLO BFKL solution in a very compact way in Eq. (60). Agreement with the NLO DGLAP anomalous dimension is established in Sec. VI. We conclude in Sec. VII.

\section{THE PROBLEM: GENERAL FORM OF THE SOLUTION OF THE ALL-ORDER BFKL EQUATION}

Our goal is to find the general form of the solution of the arbitrary-order BFKL equation

$$
\partial_{Y} G\left(k, k^{\prime}, Y\right)=\int d^{2} q K(k, q) G\left(q, k^{\prime}, Y\right)
$$

for the Green function $G\left(k, k^{\prime}, Y\right)$ with the initial condition

$$
G\left(k, k^{\prime}, Y=0\right)=\frac{1}{2 \pi k} \delta\left(k-k^{\prime}\right)
$$

Here $k \equiv\left|\vec{k}_{\perp}\right|$ and $k^{\prime} \equiv\left|\vec{k}_{\perp}^{\prime}\right|$ are the transverse momenta at the two ends of the $\operatorname{BFKL} \operatorname{ladder}, Y=\ln \left(s / k k^{\prime}\right)$ is rapidity, $s$ is the center-of-mass energy, and $\partial_{Y} \equiv \partial / \partial Y$. For simplicity we consider only the azimuthally symmetric case, in which the Green function $G$ depends only on magnitudes $k$ and $k^{\prime}$ of the two-dimensional transverse vectors $\vec{k}_{\perp}$ and $\vec{k}_{\perp}^{\prime}$ : our technique can be generalized to the case with non-trivial azimuthal-angle dependence of the scattering amplitude.

Below we will first explicitly solve the NLO BFKL equation, and then construct the general form of the solution of the all-order BFKL equation.

\section{SOLUTION OF THE NLO BFKL EQUATION}

The kernel $K(k, q)$ of the general BFKL equation (1) is known up to the next-to-leading order (NLO) [15 17, 24, 25] in the coupling constant. The general BFKL kernel can be written as

$$
K(k, q)=\bar{\alpha}_{\mu} K^{\mathrm{LO}}(k, q)+\bar{\alpha}_{\mu}^{2} K^{\mathrm{NLO}}(k, q)+\mathcal{O}\left(\bar{\alpha}_{\mu}^{3}\right)
$$


with

$$
\bar{\alpha}_{\mu} \equiv \frac{\alpha_{\mu} N_{c}}{\pi}
$$

(Here $\alpha_{\mu}$ is the renormalized strong coupling constant at an arbitrary renormalization scale $\mu$.)

The exact form of the leading-order (LO) and NLO kernels $K^{\mathrm{LO}}$ and $K^{\mathrm{NLO}}$ will not be needed below (see 15 17 and [24, 25] correspondingly). However, it is essential for us to know the action of the LO and NLO BFKL kernels on the eigenfunctions of the LO kernel [24, 25]:

$$
\int d^{2} q K^{\mathrm{LO}+\mathrm{NLO}}(k, q) q^{2 \gamma-2}=\left[\bar{\alpha}_{\mu} \chi_{0}(\gamma)-\bar{\alpha}_{\mu}^{2} \beta_{2} \chi_{0}(\gamma) \ln \frac{k^{2}}{\mu^{2}}+\bar{\alpha}_{\mu}^{2} \frac{\delta(\gamma)}{4}\right] k^{2 \gamma-2}
$$

where

$$
K^{\mathrm{LO}+\mathrm{NLO}}(k, q) \equiv \bar{\alpha}_{\mu} K^{\mathrm{LO}}(k, q)+\bar{\alpha}_{\mu}^{2} K^{\mathrm{NLO}}(k, q)
$$

is a shorthand notation for the sum of the LO and NLO kernels. In Eq. (5) we employed the eigenvalue of the LO BFKL kernel

$$
\chi_{0}(\gamma)=2 \psi(1)-\psi(\gamma)-\psi(1-\gamma)
$$

with $\psi(\gamma)=\Gamma^{\prime}(\gamma) / \Gamma(\gamma)$. The coefficient $\beta_{2}$ determines the one-loop QCD beta-function [41, 50], such that the corresponding one-loop QCD running coupling constant is given by

$$
\bar{\alpha}_{s}\left(Q^{2}\right)=\frac{\bar{\alpha}_{\mu}}{1+\bar{\alpha}_{\mu} \beta_{2} \ln \frac{Q^{2}}{\mu^{2}}}, \quad \beta_{2}=\frac{11 N_{c}-2 N_{f}}{12 N_{c}} .
$$

In Eq. (5]) we are following the notation of Fadin and Lipatov [24]: the function $\delta(\gamma)$ is rather involved and is given by Eq. (14) in [24] (for our definition of rapidity, $Y=\ln \left(s / k k^{\prime}\right)$ ).

For future use we need to separate in Eq. (5) the terms symmetric and anti-symmetric under the $\gamma \leftrightarrow 1-\gamma$ interchange. While $\chi_{0}(\gamma)$ is explicitly symmetric under such interchange, $\delta(\gamma)$ can be decomposed as [24]

$$
\frac{\delta(\gamma)}{4}=-\frac{1}{2} \beta_{2} \chi_{0}^{\prime}(\gamma)+\chi_{1}(\gamma)
$$

where $\chi_{0}^{\prime}(\gamma)=\frac{d}{d \gamma} \chi_{0}(\gamma)$ generates the anti-symmetric under $\gamma \leftrightarrow 1-\gamma$ term and $\chi_{1}(\gamma)$ denotes the symmetric term and is given by 24 ]

$$
\begin{aligned}
\chi_{1}(\gamma)= & -\beta_{2} \frac{\chi_{0}^{2}(\gamma)}{2}+\frac{5}{3} \beta_{2} \chi_{0}(\gamma)+\frac{1}{3}\left(1-\frac{\pi^{2}}{4}\right) \chi_{0}(\gamma)+\frac{3}{2} \zeta(3) \\
& -\frac{\pi^{2} \cos (\pi \gamma)}{4 \sin ^{2}(\pi \gamma)(1-2 \gamma)}\left[3+\left(1+\frac{N_{f}}{N_{c}^{3}}\right) \frac{2+3 \gamma-3 \gamma^{2}}{3+4 \gamma-4 \gamma^{2}}\right]-\frac{1}{4} \chi_{0}^{\prime \prime}(\gamma)+\frac{\pi^{3}}{4 \sin (\pi \gamma)}-\phi(\gamma)
\end{aligned}
$$

with $\zeta(z)$ the Riemann $\zeta$-function, $\chi_{0}^{\prime \prime}(\gamma)=\frac{d^{2}}{d \gamma^{2}} \chi_{0}(\gamma)$, and

$$
\phi(\gamma)=\sum_{n=0}^{\infty}(-1)^{n}\left[\frac{\psi(n+1+\gamma)-\psi(1)}{(n+\gamma)^{2}}+\frac{\psi(n+2-\gamma)-\psi(1)}{(n+1-\gamma)^{2}}\right] .
$$

Using the decomposition (9) we rewrite Eq. (5) as

$$
\int d^{2} q K^{\mathrm{LO}+\mathrm{NLO}}(k, q) q^{2 \gamma-2}=\left[\bar{\alpha}_{\mu} \chi_{0}(\gamma)-\bar{\alpha}_{\mu}^{2} \beta_{2} \chi_{0}(\gamma) \ln \frac{k^{2}}{\mu^{2}}-\frac{1}{2} \bar{\alpha}_{\mu}^{2} \beta_{2} \chi_{0}^{\prime}(\gamma)+\bar{\alpha}_{\mu}^{2} \chi_{1}(\gamma)\right] k^{2 \gamma-2}
$$

It is clear from Eq. (5) (or from Eq. (12) ) that the eigenfunctions of the LO BFKL kernel (simple powers $k^{2 \gamma-2}$ in the azimuthally symmetric case at hand) are not the eigenfunctions of the NLO kernel, since the expression in the square brackets on the right-hand-side of Eq. (5) is also $k$-dependent. Notice also that this property is entirely due to the running of the coupling constant (and this is why the $k$-dependence in the square brackets on the right of Eq. (5) comes in with a factor of $\beta_{2}$ ): in the conformal $\mathcal{N}=4 \mathrm{SYM}$ theory, where the coupling does not run, eigenfunctions of the LO BFKL kernel are also eigenfunctions of the arbitrary-order kernel, such that construction of the general form of the solution is rather straightforward [45 48]. 


\section{A. Constructing the Eigenfunctions}

As emphasized in the Introduction, we will find a set of eigenfunctions for the LO+NLO BFKL kernel. Since $K^{\mathrm{LO}+\mathrm{NLO}}$ is a perturbative expansion of the full BFKL equation kernel, it appears logical to attempt constructing the LO+NLO BFKL kernel's eigenfunctions perturbatively as well, order by order in the coupling constant. The LO BFKL kernel eigenfunctions are known: these are simply $k^{2 \gamma-2}$. We will look for the LO+NLO BFKL kernel eigenfunctions as perturbative corrections to this LO eigenfunctions. We thus write the LO+NLO eigenfunctions as

$$
H_{\gamma}(k)=k^{2 \gamma-2}+\bar{\alpha}_{\mu} F_{\gamma}(k)
$$

where the $F_{\gamma}(k)$ is a function to be determined in order to make $H_{\gamma}(k)$ the eigenfunctions of the LO+NLO kernel.

Acting on $H_{\gamma}(k)$ with the LO+NLO kernel and employing Eq. (12), within the order- $\bar{\alpha}_{\mu}$ accuracy we have

$$
\begin{aligned}
& \int d^{2} q\left[K^{\mathrm{LO}}(k, q)+\bar{\alpha}_{\mu} K^{\mathrm{NLO}}(k, q)\right] H_{\gamma}(q) \\
& =\chi_{0}(\gamma) k^{2 \gamma-2}+\bar{\alpha}_{\mu} \int d^{2} q K^{\mathrm{LO}}(k, q) F_{\gamma}(q)-\bar{\alpha}_{\mu} \beta_{2} \chi_{0}(\gamma) k^{2 \gamma-2} \ln \frac{k^{2}}{\mu^{2}}+\bar{\alpha}_{\mu}\left(-\frac{1}{2} \beta_{2} \chi_{0}^{\prime}(\gamma)+\chi_{1}(\gamma)\right) H_{\gamma}(k) \\
& =\chi_{0}(\gamma) H_{\gamma}(k)-\bar{\alpha}_{\mu} \chi_{0}(\gamma) F_{\gamma}(k)+\bar{\alpha}_{\mu} \int d^{2} q K^{\mathrm{LO}}(q, k) F_{\gamma}(q)-\bar{\alpha}_{\mu} \beta_{2} \chi_{0}(\gamma) k^{2 \gamma-2} \ln \frac{k^{2}}{\mu^{2}} \\
& \quad+\bar{\alpha}_{\mu}\left(-\frac{1}{2} \beta_{2} \chi_{0}^{\prime}(\gamma)+\chi_{1}(\gamma)\right) H_{\gamma}(k) .
\end{aligned}
$$

From (14) we deduce that $H_{\gamma}(k)$ is an eigenfunction of the LO+NLO kernel if there exists a function $c(\gamma)$ such that (up to order- $\bar{\alpha}_{\mu}$ corrections)

$$
{ }_{-} \chi_{0}(\gamma) F_{\gamma}(k)+\int d^{2} q K^{\mathrm{LO}}(k, q) F_{\gamma}(q)-\beta_{2} \chi_{0}(\gamma) H_{\gamma}(k) \ln \frac{k^{2}}{\mu^{2}}=c(\gamma) H_{\gamma}(k)
$$

so that we would have

$$
\int d^{2} q K^{\mathrm{LO}+\mathrm{NLO}}(k, q) H_{\gamma}(q)=\left[\bar{\alpha}_{\mu} \chi_{0}(\gamma)+\bar{\alpha}_{\mu}^{2}\left(-\frac{1}{2} \beta_{2} \chi_{0}^{\prime}(\gamma)+\chi_{1}(\gamma)+c(\gamma)\right)\right] H_{\gamma}(k) \equiv \Delta(\gamma) H_{\gamma}(k)
$$

Here we have also defined a general notation, $\Delta(\gamma)$, for the eigenvalue of the BFKL kernel. Our task is to determine the functions $c(\gamma)$ and $F_{\gamma}(k)$.

At the order we are working we can rewrite (15) as

$$
-\chi_{0}(\gamma) F_{\gamma}(k)+\int d^{2} q K^{\mathrm{LO}}(k, q) F_{\gamma}(q)-\beta_{2} \chi_{0}(\gamma) k^{2 \gamma-2} \ln \frac{k^{2}}{\mu^{2}}=c(\gamma) k^{2 \gamma-2}
$$

and deduce that $F_{\gamma}(k)$ has to be proportional to $k^{2 \gamma-2}$. Equation (17) gives the condition that $F_{\gamma}(k)$ has to satisfy in order for $H_{\gamma}(k)$ be an eigenfunction of the NLO kernel. We look for a solution of (17) using the following ansatz

$$
F_{\gamma}(k)=\sum_{n=0}^{\infty} c_{n}(\gamma)\left(\ln \frac{k^{2}}{\mu^{2}}\right)^{n} k^{2 \gamma-2}
$$

where $c(\gamma)$ are some (smooth) complex-valued functions of $\gamma$. Substituting Eq. (18) into Eq. (17) and dividing everything by $k^{2 \gamma-2}$ yields

$$
-\chi_{0}(\gamma) \sum_{n=0}^{\infty} c_{n}(\gamma)\left(\ln \frac{k^{2}}{\mu^{2}}\right)^{n}+\sum_{n=0}^{\infty} c_{n}(\gamma) k^{-2 \gamma+2} \int d^{2} q K^{\mathrm{LO}}(k, q)\left(\ln \frac{q^{2}}{\mu^{2}}\right)^{n} q^{2 \gamma-2}=c(\gamma)+\beta_{2} \chi_{0}(\gamma) \ln \frac{k^{2}}{\mu^{2}}
$$

Rewriting the logarithms as derivatives with respect to $\gamma$ in the second term on the left of Eq. (19) we get

$$
\sum_{n=0}^{\infty} c_{n}(\gamma) \sum_{m=0}^{n-1}\left(\begin{array}{c}
n \\
m
\end{array}\right) \chi_{0}^{(n-m)}(\gamma) \ln ^{m} \frac{k^{2}}{\mu^{2}}=c(\gamma)+\beta_{2} \chi_{0}(\gamma) \ln \frac{k^{2}}{\mu^{2}}
$$

where $\chi_{0}^{(n)}(\gamma)=d^{n} \chi_{0}(\gamma) / d \gamma^{n}$. 
To find the solution of (20) we truncate the series (18) at $n=2$, that is we put $c_{3}(\gamma)=c_{4}(\gamma)=c_{5}(\gamma)=\ldots=0$, leaving only $c_{0}(\gamma), c_{1}(\gamma)$, and $c_{2}(\gamma)$ non-zero. As we will shortly see, this truncation allows us to find a solution for $F_{\gamma}(k)$, that is a set of eigenfunctions for the LO+NLO BFKL kernel. Note that our goal is to find a complete ortho-normal set of eigenfunctions of $K^{L O+N L O}$. Therefore it does not matter whether our technique of finding the solution of (20) is general enough as long as a complete set of eigenfunctions is found.

Keeping only $c_{0}(\gamma), c_{1}(\gamma)$, and $c_{2}(\gamma)$ non-zero in Eq. (20) we obtain

$$
c_{1}(\gamma) \chi_{0}^{\prime}(\gamma)+c_{2}(\gamma)\left[\chi_{0}^{\prime \prime}(\gamma)+2 \chi_{0}^{\prime}(\gamma) \ln \frac{k^{2}}{\mu^{2}}\right]=c(\gamma)+\beta_{2} \chi_{0}(\gamma) \ln \frac{k^{2}}{\mu^{2}}
$$

Since Eq. (21) has to be valid at all values of $k$, we equate the coefficients of the terms proportional to $\ln \frac{k^{2}}{\mu^{2}}$ on both sides of (21) to get

$$
c_{2}(\gamma)=\frac{\beta_{2} \chi_{0}(\gamma)}{2 \chi_{0}^{\prime}(\gamma)}
$$

The remaining terms in (21) (without $\left.\ln \frac{k^{2}}{\mu^{2}}\right)$ give

$$
c(\gamma)=c_{1}(\gamma) \chi_{0}^{\prime}(\gamma)+\frac{\beta_{2} \chi_{0}(\gamma) \chi_{0}^{\prime \prime}(\gamma)}{2 \chi_{0}^{\prime}(\gamma)}
$$

Substituting $c_{2}$ from (22) along with $c_{3}=c_{4}=\ldots=0$ into Eqs. (18) and (13) we obtain the eigenfunctions of the $\mathrm{LO}+\mathrm{NLO}$ BFKL kernel,

$$
H_{\gamma}(k)=k^{2 \gamma-2}\left[1+\bar{\alpha}_{\mu}\left(\frac{\beta_{2} \chi_{0}(\gamma)}{2 \chi_{0}^{\prime}(\gamma)} \ln ^{2} \frac{k^{2}}{\mu^{2}}+c_{1}(\gamma) \ln \frac{k^{2}}{\mu^{2}}+c_{0}(\gamma)\right)\right],
$$

for any regular and smooth complex-valued functions $c_{0}(\gamma)$ and $c_{1}(\gamma)$. The corresponding eigenvalues $\Delta(\gamma)$ can be read off from Eqs. (16) and (23).

It is worth noticing that although we searched for a correction to the eigenfunction in $\bar{\alpha}_{\mu}$, we ended up automatically with a correction proportional to $\bar{\alpha}_{\mu} \beta_{2}$. This is indicative of the fact that we are actually expanding around the conformal point. This is not surprising, since the terms breaking conformal invariance and therefore making the LO BFKL kernel eigenfunctions not eigenfunctions of the NLO BFKL kernel, are proportional to $\beta_{2}$. Hence it is natural to expect that the LO eigenfunction gets corrections at NLO proportional to $\beta_{2}$.

Since $c_{0}(\gamma)$ and $c_{1}(\gamma)$ are arbitrary smooth functions of $\gamma$ we have a set of eigenfunctions in Eq. (24) for each $c_{0}(\gamma)$ and $c_{1}(\gamma)$. By requiring that the eigenfunctions $H_{\gamma}(k)$ satisfy the completeness and orthogonality relations at order $\alpha_{\mu}$ would reduce the freedom in these functions by generating constraints on $c_{1}(\gamma)$ and $/$ or $c_{0}(\gamma)$.

\section{B. Completeness and Orthogonality}

The completeness relation for the $H_{\gamma}(k)$ eigenfunctions is

$$
\int_{\sigma-i \infty}^{\sigma+i \infty} \frac{d \gamma}{2 \pi i} H_{\gamma}(k) H_{\gamma}^{*}\left(k^{\prime}\right)=\delta\left(k^{2}-k^{2}\right)
$$

where $\sigma$ is a real parameter that has to be determined so that Eq. (25) is satisfied and the asterisk denotes complex conjugation. We need to substitute $H_{\gamma}(k)$ from Eq. (24) into Eq. (25) and make sure the latter is satisfied order-byorder in $\bar{\alpha}_{\mu}$.

The leading-order term in Eq. (24) already satisfies Eq. (25) for

$$
\gamma=\frac{1}{2}+i \nu
$$

with $\nu$ a real variable. This fixes $\sigma=\frac{1}{2}$, such that Eq. 25) becomes

$$
\int_{\frac{1}{2}-i \infty}^{\frac{1}{2}+i \infty} \frac{d \gamma}{2 \pi i} H_{\gamma}(k) H_{\gamma}^{*}\left(k^{\prime}\right)=\delta\left(k^{2}-k^{2}\right) .
$$


Note that at LO the power-law functions $k^{2 \gamma-2}$ are eigenfunctions of the LO BFKL kernel for any $\gamma$ (with $0<$ $\operatorname{Re} \gamma<1)$. However for $\gamma=\frac{1}{2}+i \nu$ with $\nu$ a real parameter, these power-law functions form an ortho-normal set in the functional space (of LO BFKL solutions) upon which the action of the LO kernel is well-defined and yields normalized functions. (That is, the integrals like

$$
\int d^{2} k d^{2} q f^{*}(k) K(k, q) g(q)
$$

are finite for any functions $f(k), g(k)$ from the set.) Consequently, the LO BFKL kernel acts on powers $k^{-1+2 i \nu}$ as a hermitean operator with real eigenvalues. Hermiticity of the BFKL kernel has a straightforward physical interpretation: BFKL ladder (drawn vertically) is up-down symmetric. Formally this means that the BFKL kernel satisfies the following property $K(k, q)=K(q, k)$ both at LO and at NLO 15 17, 24, 25], which, when combined with the fact that the kernel is real, implies that it is hermitean. One therefore expects that the eigenvalues of the BFKL kernel for $\gamma=\frac{1}{2}+i \nu$ should be real to any order in the coupling constant. For the LO eigenvalues (7) taken at $\gamma=\frac{1}{2}+i \nu$ this property is obviously satisfied:

$$
\chi_{0}(\nu) \equiv \chi_{0}\left(\gamma=\frac{1}{2}+i \nu\right)=2 \psi(1)-\psi\left(\frac{1}{2}+i \nu\right)-\psi\left(\frac{1}{2}-i \nu\right)
$$

is manifestly real for real $\nu$. We will shortly see that the reality condition is satisfied by the NLO eigenvalues from Eq. (16).

In the following, it will be more convenient to keep track of the real and imaginary parts of the expressions by switching to the $\nu$ variable using Eq. (26). To that end we rewrite Eq. (24) as

$$
H_{\frac{1}{2}+i \nu}(k)=k^{-1+2 i \nu}\left[1+\bar{\alpha}_{\mu}\left(i \frac{\beta_{2} \chi_{0}(\nu)}{2 \chi_{0}^{\prime}(\nu)} \ln ^{2} \frac{k^{2}}{\mu^{2}}+c_{1}(\nu) \ln \frac{k^{2}}{\mu^{2}}+c_{0}(\nu)\right)\right],
$$

where we defined $c_{j}(\nu) \equiv c_{j}(\gamma(\nu))=\operatorname{Re}\left[c_{j}(\nu)\right]+i \operatorname{Im}\left[c_{j}(\nu)\right]$ for $j=0,1$ and $\chi_{0}^{\prime}(\nu)=d \chi_{0}(\nu) / d \nu$.

Substituting (30) into (27) we obtain the following condition at order- $\bar{\alpha}_{\mu}$ :

$$
\int_{-\infty}^{\infty} d \nu\left(\frac{k^{2}}{k^{\prime 2}}\right)^{i \nu}\left[i \frac{\beta_{2} \chi_{0}(\nu)}{\chi_{0}^{\prime}(\nu)} \ln \frac{k k^{\prime}}{\mu^{2}} \ln \frac{k^{2}}{k^{\prime 2}}+2 \operatorname{Re}\left[c_{1}(\nu)\right] \ln \frac{k k^{\prime}}{\mu^{2}}+i \operatorname{Im}\left[c_{1}(\nu)\right] \ln \frac{k^{2}}{k^{\prime 2}}+2 \operatorname{Re}\left[c_{0}(\nu)\right]\right]=0 .
$$

The first thing to notice in Eq. (31) is that $\operatorname{Im}\left[c_{0}(\nu)\right]$ does not get constrained by the completeness relation since it cancels out in the $H_{\frac{1}{2}+i \nu}(k)\left[H_{\frac{1}{2}+i \nu}\left(k^{\prime}\right)\right]^{*}$ product at order- $\bar{\alpha}_{\mu}$. From (31) it is also clear that the terms that are $\mu$-dependent should vanish independently from the rest: ${ }^{1}$ this gives

$$
0=\int_{-\infty}^{\infty} d \nu\left(\frac{k^{2}}{k^{\prime 2}}\right)^{i \nu}\left[i \frac{\beta_{2} \chi_{0}(\nu)}{\chi_{0}^{\prime}(\nu)} \ln \frac{k^{2}}{k^{\prime 2}}+2 \operatorname{Re}\left[c_{1}(\nu)\right]\right]=\int_{-\infty}^{\infty} d \nu\left(\frac{k^{2}}{k^{\prime 2}}\right)^{i \nu}\left[-\frac{\partial}{\partial \nu}\left(\frac{\beta_{2} \chi_{0}(\nu)}{\chi_{0}^{\prime}(\nu)}\right)+2 \operatorname{Re}\left[c_{1}(\nu)\right]\right] .
$$

Eq. (32) is satisfied for all $k$ and $k^{\prime}$ only if

$$
\operatorname{Re}\left[c_{1}(\nu)\right]=\frac{\beta_{2}}{2} \frac{\partial}{\partial \nu}\left(\frac{\chi_{0}(\nu)}{\chi_{0}^{\prime}(\nu)}\right)=\frac{\beta_{2}}{2}\left(1-\frac{\chi_{0}(\nu) \chi_{0}^{\prime \prime}(\nu)}{\chi_{0}^{\prime}(\nu)^{2}}\right) .
$$

We have determined $\operatorname{Re}\left[c_{1}(\nu)\right]$. Notice that the integral in (32) contains a divergence at $\nu=0$ due to $\chi_{0}^{\prime}(\nu)$ in the denominator of the first term: we assume that the divergence is regularized using the principle value prescription. Now, using (33) in (31) we have

$$
0=\int_{-\infty}^{\infty} d \nu\left(\frac{k^{2}}{k^{\prime 2}}\right)^{i \nu}\left[i \operatorname{Im}\left[c_{1}(\nu)\right] \ln \frac{k^{2}}{k^{\prime 2}}+2 \operatorname{Re}\left[c_{0}(\nu)\right]\right]=\int_{-\infty}^{\infty} d \nu\left(\frac{k^{2}}{k^{\prime 2}}\right)^{i \nu}\left[-\frac{\partial}{\partial \nu}\left(\operatorname{Im}\left[c_{1}(\nu)\right]\right)+2 \operatorname{Re}\left[c_{0}(\nu)\right]\right]
$$

\footnotetext{
${ }^{1}$ Since $c_{1}(\nu)$ and $c_{0}(\nu)$ are dimensionless and momentum-independent, they can not depend on $\mu$.
} 
which is satisfied for all $k$ and $k^{\prime}$ only if

$$
-\frac{\partial}{\partial \nu}\left(\operatorname{Im}\left[c_{1}(\nu)\right]\right)+2 \operatorname{Re}\left[c_{0}(\nu)\right]=0
$$

We see that the completeness relation (25) is not sufficient to completely fix $c_{0}(\nu)\left(\right.$ with $\operatorname{Im}\left[c_{1}(\nu)\right]$ fixed by $\operatorname{Re}\left[c_{0}(\nu)\right]$ through (35).

Keeping this in mind, and using Eqs. (33) and (35) in Eq. (30) we arrive at the following form of the LO+NLO BFKL kernel eigenfunctions

$$
H_{\frac{1}{2}+i \nu}(k)=k^{-1+2 i \nu}\left[1+\bar{\alpha}_{\mu}\left(i \frac{\beta_{2} \chi_{0}(\nu)}{2 \chi_{0}^{\prime}(\nu)} \ln ^{2} \frac{k^{2}}{\mu^{2}}+\frac{\beta_{2}}{2}\left(1-\frac{\chi_{0}(\nu) \chi_{0}^{\prime \prime}(\nu)}{\chi_{0}^{\prime}(\nu)^{2}}\right) \ln \frac{k^{2}}{\mu^{2}}+i \operatorname{Im}\left[c_{1}(\nu)\right] \ln \frac{k^{2}}{\mu^{2}}+c_{0}(\nu)\right)\right] .
$$

The eigenvalues $\Delta(\nu) \equiv \Delta(\gamma(\nu))$ of the eigenfunctions (36) can be easily constructed using Eqs. (16) and (23):

$$
\begin{aligned}
\Delta(\nu) & =\bar{\alpha}_{\mu} \chi_{0}(\nu)+\bar{\alpha}_{\mu}^{2}\left(\frac{i}{2} \beta_{2} \chi_{0}^{\prime}(\nu)+\chi_{1}(\nu)+c(\nu)\right) \\
& =\bar{\alpha}_{\mu} \chi_{0}(\nu)+\bar{\alpha}_{\mu}^{2}\left(\frac{i}{2} \beta_{2} \chi_{0}^{\prime}(\nu)+\chi_{1}(\nu)-i c_{1}(\nu) \chi_{0}^{\prime}(\nu)-i \frac{\beta_{2} \chi_{0}(\nu) \chi_{0}^{\prime \prime}(\nu)}{2 \chi_{0}^{\prime}(\nu)}\right) .
\end{aligned}
$$

Here $\chi_{1}(\nu) \equiv \chi_{1}(\gamma(\nu))$ and $c(\nu) \equiv c(\gamma(\nu))$. Finally, with the help of (33) we rewrite Eq. (37) as

$$
\Delta(\nu)=\bar{\alpha}_{\mu} \chi_{0}(\nu)+\bar{\alpha}_{\mu}^{2}\left[\chi_{1}(\nu)+\operatorname{Im}\left[c_{1}(\nu)\right] \chi_{0}^{\prime}(\nu)\right]
$$

We see that the LO+NLO eigenvalues are manifestly real, as expected from eigenvalues of a hermitean operator such as the LO+NLO BFKL kernel! Note also that the reality of the eigenvalues was achieved after the cancellation of the $(\gamma \leftrightarrow 1-\gamma)$-odd term from Eq. (9]) calculated in [24]: as $\gamma \leftrightarrow 1-\gamma$ corresponds to $\nu \leftrightarrow-\nu$, this term is in fact imaginary, and it had to cancel for us to obtain a real eigenvalue.

Eqs. (36) and (38) give us the LO+NLO BFKL kernel eigenfunctions and their corresponding eigenvalues up to a freedom of choosing $c_{0}(\nu)$ (with $\operatorname{Im}\left[c_{1}(\nu)\right]$ fixed by (35)). Below we will show that the solution of LO+NLO BFKL equation does not depend on $c_{0}(\nu)$ (and $\operatorname{Im}\left[c_{1}(\nu)\right]$ ), such that one is always free to put them to zero (both in the eigenfunctions (36) and in the eigenvalues (38) ) and no ambiguity is left.

In Appendix A we show that the eigenfunctions (36) also satisfy the orthogonality condition

$$
\int d^{2} k H_{\frac{1}{2}+i \nu}(k)\left[H_{\frac{1}{2}+i \nu^{\prime}}(k)\right]^{*}=2 \pi^{2} \delta\left(\nu-\nu^{\prime}\right) .
$$

Note that orthogonality does not impose any additional constraints on $c_{0}(\nu)$, thus leaving it unspecified.

\section{The Phase and $\nu$-Reparametrization Freedom}

Before we clarify the origin of the remaining freedom of choosing $c_{0}(\nu)$ in the eigenfunctions (36), let us note that we have found eigenfunctions of the LO+NLO BFKL kernel satisfying the completeness (27) and orthogonality (39) conditions: however, these conditions do not yet fix the eigenfunctions uniquely. In this Subsection we will show that there are two trivial degrees of freedom in the choice of eigenfunctions, one due to a choice of the overall phase, while another one is due to a choice of the variable $\nu$. These symmetries of the problem will allow us to always choose $\mathrm{LO}+\mathrm{NLO}$ BFKL eigenfunctions with $c_{0}(\nu)=0$ and $\operatorname{Im}\left[c_{1}(\nu)\right]=0$.

An eigenfunction of BFKL kernel remains an eigenfunction after a rescaling by a constant. Eq. (39), being an ortho-normality condition, limits the rescaling freedom for functions $H_{\frac{1}{2}+i \nu}(k)$ to

$$
H_{\frac{1}{2}+i \nu}(k) \rightarrow e^{i \theta} H_{\frac{1}{2}+i \nu}(k)
$$

with any real phase $\theta$. Note that the same phase freedom exists already in the LO BFKL eigenfunctions, since we can always rescale $k^{-1+2 i \nu} \rightarrow e^{i \theta} k^{-1+2 i \nu}$. The phase $\theta$ is fixed by convention: at the LO it is usually put to zero. Similarly at NLO we can always remove the phase of $H_{\frac{1}{2}+i \nu}(k)$ by rescaling

$$
H_{\frac{1}{2}+i \nu}(k) \rightarrow e^{-i \bar{\alpha}_{\mu}\left(\operatorname{Im}\left[c_{0}(\nu)\right]-\operatorname{Im}\left[c_{1}(\nu)\right] \ln \mu^{2}\right)} H_{\frac{1}{2}+i \nu}(k) \approx\left[1-i \bar{\alpha}_{\mu}\left(\operatorname{Im}\left[c_{0}(\nu)\right]-\operatorname{Im}\left[c_{1}(\nu)\right] \ln \mu^{2}\right)\right] H_{\frac{1}{2}+i \nu}(k)
$$


That is we can always choose the phase convention in which $\operatorname{Im}\left[c_{0}(\nu)\right]$ is zero and the LO+NLO BFKL eigenfunctions are

$$
H_{\frac{1}{2}+i \nu}(k)=k^{-1+2 i \nu}\left[1+\bar{\alpha}_{\mu}\left(i \frac{\beta_{2} \chi_{0}(\nu)}{2 \chi_{0}^{\prime}(\nu)} \ln ^{2} \frac{k^{2}}{\mu^{2}}+\frac{\beta_{2}}{2}\left(1-\frac{\chi_{0}(\nu) \chi_{0}^{\prime \prime}(\nu)}{\chi_{0}^{\prime}(\nu)^{2}}\right) \ln \frac{k^{2}}{\mu^{2}}+i \operatorname{Im}\left[c_{1}(\nu)\right] \ln k^{2}+\operatorname{Re}\left[c_{0}(\nu)\right]\right)\right]
$$

with the eigenvalues still given by Eq. (38). ${ }^{2}$

Let us show now how to eliminate $\operatorname{Im}\left[c_{1}(\nu)\right]$ and $\operatorname{Re}\left[c_{0}(\nu)\right]$ in Eq. (42) by using another degree of freedom, namely the choice of reparametrization for the variable $\nu$ labeling the eigenfunctions. For instance, let us shift the $\nu$-variable by defining

$$
\nu^{\prime}=\nu+\bar{\alpha}_{\mu} \operatorname{Im}\left[c_{1}(\nu)\right]
$$

in the completeness relation (27) with the $H$-function given in (42). We obtain (to order- $\bar{\alpha}_{\mu}$ )

$$
\begin{aligned}
\delta\left(k^{2}-k^{\prime 2}\right)= & \int_{-\infty}^{+\infty} \frac{d \nu}{2 \pi} H_{\frac{1}{2}+i \nu}(k)\left[H_{\frac{1}{2}+i \nu}\left(k^{\prime}\right)\right]^{*} \\
= & \int_{-\infty}^{+\infty} \frac{d \nu^{\prime}}{2 \pi} \frac{d \nu}{d \nu^{\prime}} \frac{1}{k k^{\prime}}\left(\frac{k^{2}}{k^{\prime 2}}\right)^{i \nu^{\prime}}\left(1-i \bar{\alpha}_{\mu} \operatorname{Im}\left[c_{1}\left(\nu^{\prime}\right)\right] \ln k^{2}\right)\left(1+i \bar{\alpha}_{\mu} \operatorname{Im}\left[c_{1}\left(\nu^{\prime}\right)\right] \ln k^{\prime 2}\right) \\
& \times\left[1+\bar{\alpha}_{\mu}\left(i \frac{\beta_{2} \chi_{0}\left(\nu^{\prime}\right)}{2 \chi_{0}^{\prime}\left(\nu^{\prime}\right)} \ln ^{2} \frac{k^{2}}{\mu^{2}}+\frac{\beta_{2}}{2}\left(1-\frac{\chi_{0}\left(\nu^{\prime}\right) \chi_{0}^{\prime \prime}\left(\nu^{\prime}\right)}{\chi_{0}^{\prime}\left(\nu^{\prime}\right)^{2}}\right) \ln \frac{k^{2}}{\mu^{2}}+i \operatorname{Im}\left[c_{1}\left(\nu^{\prime}\right)\right] \ln k^{2}+\operatorname{Re}\left[c_{0}\left(\nu^{\prime}\right)\right]\right)\right] \\
& \times\left[1+\bar{\alpha}_{\mu}\left(-i \frac{\beta_{2} \chi_{0}\left(\nu^{\prime}\right)}{2 \chi_{0}^{\prime}\left(\nu^{\prime}\right)} \ln ^{2} \frac{k^{\prime 2}}{\mu^{2}}+\frac{\beta_{2}}{2}\left(1-\frac{\chi_{0}\left(\nu^{\prime}\right) \chi_{0}^{\prime \prime}\left(\nu^{\prime}\right)}{\chi_{0}^{\prime}\left(\nu^{\prime}\right)^{2}}\right) \ln \frac{k^{\prime 2}}{\mu^{2}}-i \operatorname{Im}\left[c_{1}\left(\nu^{\prime}\right)\right] \ln k^{\prime 2}+\operatorname{Re}\left[c_{0}\left(\nu^{\prime}\right)\right]\right)\right] .
\end{aligned}
$$

Using

$$
\frac{d \nu}{d \nu^{\prime}}=1-\bar{\alpha}_{\mu} \frac{\partial}{\partial \nu^{\prime}} \operatorname{Im}\left[c_{1}\left(\nu^{\prime}\right)\right]
$$

and the relation (35) we recast Eq. (44) as

$$
\begin{aligned}
\delta\left(k^{2}-k^{\prime 2}\right)=\int_{-\infty}^{+\infty} \frac{d \nu^{\prime}}{2 \pi} \frac{1}{k k^{\prime}}\left(\frac{k^{2}}{k^{\prime 2}}\right)^{i \nu^{\prime}} & {\left[1+\bar{\alpha}_{\mu}\left(i \frac{\beta_{2} \chi_{0}\left(\nu^{\prime}\right)}{2 \chi_{0}^{\prime}\left(\nu^{\prime}\right)} \ln ^{2} \frac{k^{2}}{\mu^{2}}+\frac{\beta_{2}}{2}\left(1-\frac{\chi_{0}\left(\nu^{\prime}\right) \chi_{0}^{\prime \prime}\left(\nu^{\prime}\right)}{\chi_{0}^{\prime}\left(\nu^{\prime}\right)^{2}}\right) \ln \frac{k^{2}}{\mu^{2}}\right)\right] } \\
\times & {\left[1+\bar{\alpha}_{\mu}\left(-i \frac{\beta_{2} \chi_{0}\left(\nu^{\prime}\right)}{2 \chi_{0}^{\prime}\left(\nu^{\prime}\right)} \ln ^{2} \frac{k^{\prime 2}}{\mu^{2}}+\frac{\beta_{2}}{2}\left(1-\frac{\chi_{0}\left(\nu^{\prime}\right) \chi_{0}^{\prime \prime}\left(\nu^{\prime}\right)}{\chi_{0}^{\prime}\left(\nu^{\prime}\right)^{2}}\right) \ln \frac{k^{\prime 2}}{\mu^{2}}\right)\right] . }
\end{aligned}
$$

Therefore, we may define the new LO+NLO BFKL eigenfunctions after the $\nu$-reparametrization (43) as

$$
H_{\frac{1}{2}+i \nu}(k)=k^{-1+2 i \nu}\left[1+\bar{\alpha}_{\mu} \beta_{2}\left(i \frac{\chi_{0}(\nu)}{2 \chi_{0}^{\prime}(\nu)} \ln ^{2} \frac{k^{2}}{\mu^{2}}+\frac{1}{2}\left(1-\frac{\chi_{0}(\nu) \chi_{0}^{\prime \prime}(\nu)}{\chi_{0}^{\prime}(\nu)^{2}}\right) \ln \frac{k^{2}}{\mu^{2}}\right)\right] .
$$

One can see from Eq. (46) that these new functions satisfy the completeness relation (27). The functions (47) may be obtained from Eq. (42) by putting $\operatorname{Im}\left[c_{1}(\nu)\right]=0$ and $\operatorname{Re}\left[c_{0}(\nu)\right]=0$ in it. This shows that functions $H_{\frac{1}{2}+i \nu}$ in (47) are the eigenfunctions of the LO+NLO BFKL kernel. We have eliminated $\operatorname{Im}\left[c_{1}(\nu)\right]$ and $\operatorname{Re}\left[c_{0}(\nu)\right]$ in (42) by simply using the freedom (43) to redefine $\nu$. One can easily see that the functions (47) also satisfy the orthogonality relation (39).

\footnotetext{
2 If one is uncomfortable with logarithms of dimensionful quantities, such as $\ln \mu^{2}$ in (41) and $\ln k^{2}$ in 42), the arguments in this Section can be repeated for dimensionless eigenfunctions defined by $\tilde{H}_{\frac{1}{2}+i \nu}(k) \equiv \mu^{1-2 i \nu} H_{\frac{1}{2}+i \nu}(k)$ if one removes $\operatorname{Im}\left[c_{1}(\nu)\right]$ from Eq. (41) while leaving Eq. (43) the same.
} 
It is also important to note that the $\nu=\nu^{\prime}-\bar{\alpha}_{\mu} \operatorname{Im}\left[c_{1}\left(\nu^{\prime}\right)\right]$ transformation eliminates $\operatorname{Im}\left[c_{1}(\nu)\right]$ from the eigenvalue (38) since, to order- $\bar{\alpha}_{\mu}$,

$$
\Delta(\nu)=\bar{\alpha}_{\mu} \chi_{0}(\nu)+\bar{\alpha}_{\mu}^{2}\left[\chi_{1}(\nu)+\operatorname{Im}\left[c_{1}(\nu)\right] \chi_{0}^{\prime}(\nu)\right]=\bar{\alpha}_{\mu} \chi_{0}\left(\nu^{\prime}\right)+\bar{\alpha}_{\mu}^{2} \chi_{1}\left(\nu^{\prime}\right)+\mathcal{O}\left(\bar{\alpha}_{\mu}^{3}\right) .
$$

We see that $\operatorname{Im}\left[c_{1}(\nu)\right]$ in the eigenvalue (38) is closely related to the choice of the $\nu$-variable, and can be eliminated by such choice.

We can always utilize the $\nu$-reparametrization invariance to remove $\operatorname{Im}\left[c_{1}(\nu)\right]$ and $\operatorname{Re}\left[c_{0}(\nu)\right]$ in Eqs. (42) and (38): note that this procedure leaves the solution unchanged. Our final form of the LO+NLO BFKL eigenfunctions $H_{\frac{1}{2}+i \nu}$ is given in Eq. (47). The corresponding eigenvalues are obtained from (38) by using $\operatorname{Im}\left[c_{1}(\nu)\right]=0$, such that

$$
\Delta(\nu)=\bar{\alpha}_{\mu} \chi_{0}(\nu)+\bar{\alpha}_{\mu}^{2} \chi_{1}(\nu) .
$$

\section{NLO BFKL Solution}

Using the eigenfunctions (47) and eigenvalues (49) of the LO+NLO BFKL kernel, along with the completeness relation (27) it is straightforward to write the solution of Eq. (11) with the initial condition (2) to the NLO accuracy as

$$
G\left(k, k^{\prime}, Y\right)=\int_{-\infty}^{\infty} \frac{d \nu}{2 \pi^{2}} e^{\left[\bar{\alpha}_{\mu} \chi_{0}(\nu)+\bar{\alpha}_{\mu}^{2} \chi_{1}(\nu)\right] Y} H_{\frac{1}{2}+i \nu}(k)\left[H_{\frac{1}{2}+i \nu}\left(k^{\prime}\right)\right]^{*} .
$$

Here $\chi_{0}(\nu)$ is given by Eq. (29) and, for completeness, we rewrite Eq. (10) in terms of the $\nu$ variable as

$$
\begin{aligned}
\chi_{1}(\nu)= & -\beta_{2} \frac{\chi_{0}^{2}(\nu)}{2}+\frac{5}{3} \beta_{2} \chi_{0}(\nu)+\frac{1}{3}\left(1-\frac{\pi^{2}}{4}\right) \chi_{0}(\nu)+\frac{3}{2} \zeta(3) \\
& -\frac{\pi^{2} \sinh (\pi \nu)}{8 \nu \cosh ^{2}(\pi \nu)}\left[3+\left(1+\frac{N_{f}}{N_{c}^{3}}\right) \frac{11+12 \nu^{2}}{16\left(1+\nu^{2}\right)}\right]+\frac{1}{4} \chi_{0}^{\prime \prime}(\nu)+\frac{\pi^{3}}{4 \cosh (\pi \nu)}-\phi\left(\frac{1}{2}+i \nu\right)
\end{aligned}
$$

with $\phi$ given by Eq. (11). The eigenfunctions $H_{\frac{1}{2}+i \nu}(k)$ are given by Eq. (47).

Eq. (50), along with Eqs. (47) and (49), are the main results of this work.

\section{GENERAL FORM OF THE SOLUTION OF HIGHER-ORDER BFKL EQUATION}

Above we have devised a solution of the NLO BFKL equation by obtaining the LO+NLO BFKL kernel eigenfunctions using a perturbative expansion around the LO conformal eigenfunctions. The resulting expression (50) contains two perturbative expansions: one in the eigenfunctions and another one in the exponent (the eigenvalue). While this is different from a solution for the DGLAP evolution equation [38 [40], which only has perturbative expansion in the anomalous dimensions (that is, in the exponent giving the power of $Q^{2}$ ), the expansions utilized in arriving at (50) are also well-defined and are under theoretical control.

Based on the success of our strategy at the NLO level, we conjecture that it can be applied to the generalized BFKL equation with the kernel calculated to an arbitrary high order in the coupling constant. The solution of the all-order BFKL equation can be formally written as

$$
G\left(k, k^{\prime}, Y\right)=\int_{-\infty}^{\infty} \frac{d \nu}{2 \pi^{2}} e^{\left[\bar{\alpha}_{\mu} \chi_{0}(\nu)+\bar{\alpha}_{\mu}^{2} \chi_{1}(\nu)+\bar{\alpha}_{\mu}^{3} \chi_{2}(\nu)+\ldots\right] Y} H_{\frac{1}{2}+i \nu}(k)\left[H_{\frac{1}{2}+i \nu}\left(k^{\prime}\right)\right]^{*}
$$

with the eigenfunctions

$$
H_{\frac{1}{2}+i \nu}(k)=k^{-1+2 i \nu}\left[1+\bar{\alpha}_{\mu} \beta_{2}\left(i \frac{\chi_{0}(\nu)}{2 \chi_{0}^{\prime}(\nu)} \ln ^{2} \frac{k^{2}}{\mu^{2}}+\frac{1}{2}\left(1-\frac{\chi_{0}(\nu) \chi_{0}^{\prime \prime}(\nu)}{\chi_{0}^{\prime}(\nu)^{2}}\right) \ln \frac{k^{2}}{\mu^{2}}\right)+\bar{\alpha}_{\mu}^{2} f_{2}\left(\frac{k}{\mu}, \nu\right)+\ldots\right] .
$$

Here $\chi_{2}(\nu)$ and higher-order coefficients indicated by the ellipsis in the exponent are the scale-invariant (conformal) $(\nu \leftrightarrow-\nu)$-even (real-valued) parts of the prefactor function generated by the action of the next-to-next-to-leadingorder (NNLO) (and higher-order) kernels on the LO eigenfunctions (cf. Eq. (12)). The function $f_{2}(k / \mu, \nu)$ denotes the NNLO corrections to the eigenfunctions, with the ellipsis in the expression (53) for the eigenfunction denoting higher-order corrections. Knowledge of the LO and NLO BFKL kernels is sufficient to construct $f_{2}(k / \mu, \nu)$, but is left for future work [51]. 


\section{PROPERTIES OF THE NLO SOLUTION}

\section{A. On $\mu$-independence of the NLO solution}

First let us note that the BFKL kernel is independent of the arbitrary renormalization scale $\mu$ at the order within the precision of the perturbative calculation [24, 25]. Since the $H_{\gamma}(k)$ eigenfunctions diagonalize the LO+NLO BFKL kernel, we may write

$$
\bar{\alpha}_{\mu} K^{\mathrm{LO}}(k, q)+\bar{\alpha}_{\mu}^{2} K^{\mathrm{NLO}}(k, q)=\int_{-\infty}^{\infty} \frac{d \nu}{2 \pi^{2}} \Delta(\nu) H_{\frac{1}{2}+i \nu}(k)\left[H_{\frac{1}{2}+i \nu}(q)\right]^{*} .
$$

From (54) it is easy to see that since the kernel on the left is $\mu$-independent, so should be the right-hand side of the expression. Iterating the action of the kernel in Eq. (54) many times we see that (in a somewhat schematic notation)

$$
\left[\bar{\alpha}_{\mu} K^{\mathrm{LO}}+\bar{\alpha}_{\mu}^{2} K^{\mathrm{NLO}}\right]^{n}(k, q)=\int_{-\infty}^{\infty} \frac{d \nu}{2 \pi^{2}} \Delta^{n}(\nu) H_{\frac{1}{2}+i \nu}(k)\left[H_{\frac{1}{2}+i \nu}(q)\right]^{*}
$$

for any positive integer $n$. Taking Eq. (27) into account we see that the integral on the right of (55) is $\mu$-independent (within the precision of the approximation, that is, up to and including order- $\bar{\alpha}_{\mu}^{n+1}$ terms) for any integer power $n \geq 0$. Expanding Eq. (50) in the powers of $Y$ we see that the coefficients of this series are proportional to the right-hand-side of Eq. (55) with $n \geq 0$, and are, therefore, $\mu$-independent.

The $\mu$-independence can be seen even more explicitly by substituting the eigenfunctions (47) into Eq. (50). This yields

$$
G\left(k, k^{\prime}, Y\right)=\int_{-\infty}^{\infty} \frac{d \nu}{2 \pi^{2} k k^{\prime}} e^{\left[\bar{\alpha}_{\mu} \chi_{0}(\nu)+\bar{\alpha}_{\mu}^{2} \chi_{1}(\nu)\right] Y}\left(\frac{k^{2}}{k^{\prime 2}}\right)^{i \nu}\left[1+\bar{\alpha}_{\mu} \beta_{2}\left(i \frac{\chi_{0}(\nu)}{\chi_{0}^{\prime}(\nu)} \ln \frac{k k^{\prime}}{\mu^{2}} \ln \frac{k^{2}}{k^{\prime 2}}+\frac{\partial}{\partial \nu}\left(\frac{\chi_{0}(\nu)}{\chi_{0}^{\prime}(\nu)}\right) \ln \frac{k k^{\prime}}{\mu^{2}}\right)\right],
$$

to order- $\bar{\alpha}_{\mu}$ in the product of the eigenfunctions. In the first term in the parenthesis of (56) we replace one of the logarithms by a derivative using

$$
\ln \frac{k^{2}}{k^{\prime 2}}\left(\frac{k^{2}}{k^{\prime 2}}\right)^{i \nu}=-i \frac{\partial}{\partial \nu}\left(\frac{k^{2}}{k^{\prime 2}}\right)^{i \nu}
$$

and integrate by parts. We arrive at

$$
G\left(k, k^{\prime}, Y\right)=\int_{-\infty}^{\infty} \frac{d \nu}{2 \pi^{2} k k^{\prime}} e^{\left[\bar{\alpha}_{\mu} \chi_{0}(\nu)+\bar{\alpha}_{\mu}^{2} \chi_{1}(\nu)\right] Y}\left(\frac{k^{2}}{k^{\prime 2}}\right)^{i \nu}\left(1-\bar{\alpha}_{\mu}^{2} \beta_{2} \chi_{0}(\nu) Y \ln \frac{k k^{\prime}}{\mu^{2}}\right)
$$

which, as can be easily verified, is $\mu$-independent up to order $\mathcal{O}\left(\bar{\alpha}_{\mu}^{3}\right)$ (cf. [52 [55]). In deriving Eq. (58), and in the calculations to follow, we employ the LLA power-counting: we assume that rapidity $Y$ is sufficiently large, such that

$$
\bar{\alpha}_{\mu} Y \sim 1,
$$

and, with this assumption, only keep order- $\bar{\alpha}_{\mu}$ terms in the prefactor. For instance, integration by parts performed in arriving at (58) also generates a term proportional to $\bar{\alpha}_{\mu}^{3} \chi_{1}^{\prime}(\nu) Y$, which is order- $\bar{\alpha}_{\mu}^{2}$ in our power-counting and, being outside the precision of our NLO approximation, is neglected. We only keep terms up to the order- $\bar{\alpha}_{\mu}$ both in the exponent and in the prefactor in the NLO approximation.

\section{B. Searching for the NNLO BFKL Solution Ansatz}

Within the precision of our NLO power-counting (using Eq. (59) and keeping only order- $\bar{\alpha}_{\mu}$ terms in the exponent and the prefactor) one could rewrite Eq. (58) as

$$
G\left(k, k^{\prime}, Y\right)=\int_{-\infty}^{\infty} \frac{d \nu}{2 \pi^{2} k k^{\prime}} e^{\left[\bar{\alpha}_{s}\left(k k^{\prime}\right) \chi_{0}(\nu)+\bar{\alpha}_{s}^{2}\left(k k^{\prime}\right) \chi_{1}(\nu)\right] Y}\left(\frac{k^{2}}{k^{\prime 2}}\right)^{i \nu}
$$


with the one-loop running coupling given by the expansion of Eq. (8) . Note that we can not uniquely fix the scale of the QCD running coupling constant at this order: in fact, we can replace the couplings in Eq. (60) by

$$
\bar{\alpha}_{s}\left(k k^{\prime}\right) \rightarrow \bar{\alpha}_{s}^{\lambda}\left(k^{2}\right) \bar{\alpha}_{s}^{\lambda}\left(k^{\prime 2}\right) \bar{\alpha}_{s}^{1-2 \lambda}\left(k k^{\prime}\right)
$$

with any real number $\lambda .{ }^{3}$ Perturbative expansions to order- $\bar{\alpha}_{\mu}^{2}$ are equivalent on the left and right sides of (61). To fix the scale(s) of the couplings in Eq. [60) one has to systematically extend the NLO BFKL solution presented above to NNLO.

The form of the solution of the NLO BFKL equation in Eq. (60) is very appealing, since it suggests an elegant way to generalize the solution of the all-order BFKL equation in $\mathcal{N}=4 \mathrm{SYM}$ theory to QCD by simply replacing the fixed couplings of $\mathcal{N}=4 \mathrm{SYM}$ theory by running QCD couplings. (Indeed the functions $\chi_{i}(\nu)$ are, in general, different in the two theories for $i \geq 1$ [45, 47, 48].)

To that end, it appears natural to suggest and test the following ansatz for the solution of the NNLO BFKL equation, which appears as a straightforward extension of Eq. (60):

$$
G\left(k, k^{\prime}, Y\right)=\int_{-\infty}^{\infty} \frac{d \nu}{2 \pi^{2} k k^{\prime}} e^{\left[\bar{\alpha}_{s}\left(k k^{\prime}\right) \chi_{0}(\nu)+\bar{\alpha}_{s}^{2}\left(k k^{\prime}\right) \chi_{1}(\nu)+\bar{\alpha}_{s}^{3}\left(k k^{\prime}\right) \chi_{2}(\nu)\right] Y}\left(\frac{k^{2}}{k^{\prime 2}}\right)^{i \nu} .
$$

As mentioned above, $\chi_{2}(\nu)$ is the $(\nu \leftrightarrow-\nu)$-even (real) scale-invariant part of the prefactor generated by the action of the NNLO BFKL kernel on the LO eigenvalue. The exact form of $\chi_{2}(\nu)$, while needed in Eq. (62), is not required to test whether the ansatz (62) in fact does solve the NNLO BFKL equation, just like we never used the exact form of $\chi_{1}(\nu)$ in constructing the NLO BFKL solution (50).

In constructing the NLO BFKL solution our knowledge of NLO kernel was defined by Eq. (12). The important to us NLO terms on its right-hand-side were the term proportional to $\ln \left(k^{2} / \mu^{2}\right)$ and the term containing $\chi_{0}^{\prime}(\nu)$. The latter term became complex for $\gamma=\frac{1}{2}+i \nu$ and was canceled to give a real eigenvalue (49). The term proportional to $\ln \left(k^{2} / \mu^{2}\right)$ resulted from the running of the coupling in the LO BFKL eigenvalue: it is clear that with $k$ being the only momentum scale left on the right of Eq. (12) the coupling there has to run with the scale $k^{2}$. One may conclude that the $k$-dependent terms appearing after the action of the NNLO kernel on the LO eigenfunction are entirely due to the running of the couplings in the LO and NLO terms with the scale $k^{2}$. We can, therefore, write for the action of the NNLO kernel on the LO eigenvalues as

$$
\begin{array}{r}
\int d^{2} q K^{\mathrm{LO}+\mathrm{NLO}+\mathrm{NNLO}}(k, q) q^{-1+2 i \nu}=\left\{\bar{\alpha}_{\mu} \chi_{0}(\nu)\left[1-\bar{\alpha}_{\mu} \beta_{2} \ln \frac{k^{2}}{\mu^{2}}+\bar{\alpha}_{\mu}^{2} \beta_{2}^{2} \ln ^{2} \frac{k^{2}}{\mu^{2}}+\bar{\alpha}_{\mu}^{2} \beta_{3} \ln \frac{k^{2}}{\mu^{2}}\right]\right. \\
\left.+\bar{\alpha}_{\mu}^{2}\left[\frac{i}{2} \beta_{2} \chi_{0}^{\prime}(\nu)+\chi_{1}(\nu)\right]\left[1-2 \bar{\alpha}_{\mu} \beta_{2} \ln \frac{k^{2}}{\mu^{2}}\right]+\bar{\alpha}_{\mu}^{3}\left[\chi_{2}(\nu)+i \delta_{2}(\nu)\right]\right\} k^{-1+2 i \nu},
\end{array}
$$

where

$$
K^{\mathrm{LO}+\mathrm{NLO}+\mathrm{NNLO}}(k, q) \equiv \bar{\alpha}_{\mu} K^{\mathrm{LO}}(k, q)+\bar{\alpha}_{\mu}^{2} K^{\mathrm{NLO}}(k, q)+\bar{\alpha}_{\mu}^{3} K^{\mathrm{NNLO}}(k, q),
$$

$\beta_{3}$ is the two-loop coefficient in the QCD beta-function [58 60] defined by

$$
\mu^{2} \frac{d \bar{\alpha}_{\mu}}{d \mu^{2}}=-\beta_{2} \bar{\alpha}_{\mu}^{2}+\beta_{3} \bar{\alpha}_{\mu}^{3}
$$

and $i \delta_{2}(\nu)$ denotes the $(\nu \leftrightarrow-\nu)$-odd imaginary $k$-independent NNLO term on the right of (63).

While $\chi_{2}(\nu)$ and $\delta_{2}(\nu)$ are presently unknown, one may still check whether the ansatz (62) solves

$$
\partial_{Y} G\left(k, k^{\prime}, Y\right)=\int d^{2} q K^{\mathrm{LO}+\mathrm{NLO}+\mathrm{NNLO}}(k, q) G\left(q, k^{\prime}, Y\right)
$$

up to order $-\bar{\alpha}_{\mu}^{2}$ in the power-counting of (59). In Appendix B we show that this, in fact, is not the case, and the ansatz (62) does not solve the NNLO BFKL equation. ${ }^{4}$ The failure of the ansatz (62) appears to be unrelated to

\footnotetext{
${ }^{3}$ For instance, $\lambda=1 / 2$ leads to the square-root ansatz, $\sqrt{\bar{\alpha}_{s}\left(k^{2}\right) \bar{\alpha}_{s}\left(k^{\prime 2}\right)}$, while $\lambda=1$ gives a "triumvirate" structure [20, 22, 56, 57]. The running of the coupling in Eq. (60) is recovered by putting $\lambda=0$.

4 Appendix B also shows explicitly that Eq. (60) does, in fact, solve the NLO BFKL equation.
} 
the choice of the running of the coupling. Instead we propose a modified ansatz (B7) for the solution of the NNLO BFKL equation, which works only if $\delta_{2}(\nu)$ is given by Eq. (B6). Note that to verify the ansatz (B6), (B7) one has to construct NNLO eigenfunctions perturbatively, using the method presented above for the NLO calculation [51]. (While approximations for the NNLO BFKL eigenvalues exist in the literature [61, 62], our solution procedure is not designed to construct the conformal part $\chi_{2}(\nu)$ of the NNLO eigenvalue: hence construction of NNLO eigenfunctions using our method would not allow one to derive the NNLO eigenvalues explicitly (except for their imaginary parts) to be compared with [61, 62] or other works on the subject.)

\section{DGLAP ANOMALOUS DIMENSION}

In Deep Inelastic Scattering (DIS) one is interested in the Bjorken- $x$ dependence of the structure functions. It is different from the energy-dependence in, say, the up-down symmetric $\gamma^{*} \gamma^{*}$ scattering. Indeed, in the case of the up-down symmetric kernel like in the $\gamma^{*} \gamma^{*}$ scattering process, the rapidity variable $Y$ that enters the BFKL equation (1) is $Y^{\mathrm{sym}}=\ln \frac{s}{k k^{\prime}}$ used through this paper so far. In DIS we instead have $Y^{\text {DIS }}=\ln \frac{s}{k^{2}} \approx \ln \frac{1}{x}$ where $s$ is the center-of-mass energy squared of the virtual photon-hadron system, $k$ is the momentum of the reggeized gluon in the BFKL ladder that is attached to the quark anti-quark pair produced by the virtual photon. Assuming that $k \approx Q$ with $Q$ the photon virtuality, we can identify $Y^{\text {DIS }}$ with $\ln \frac{1}{x}$, where $x$ is the Bjorken scaling variable 63 .

The modification needed to account for this difference in energy dependence is irrelevant at LO but it becomes important at NLO accuracy. As explained in [24], in DIS case one has to modify the evolution kernel by adding an extra term to it that consequently introduces a new additive correction to the "eigenvalues" which is proportional to $\bar{\alpha}_{\mu}^{2} \chi_{0}(\gamma) \chi_{0}^{\prime}(\gamma)$

We will now show that our solution of the BFKL equation in the symmetric case given by (50) leads to the same term in the eigenvalue for DIS: the term is generated by the change in the definition of rapidity, without having to solve the eigenvalue problem again. In this way one recovers exactly the term proportional to $\bar{\alpha}_{\mu}^{2} \chi_{0}(\gamma) \chi_{0}^{\prime}(\gamma)$ that was found in [24].

Writing $Y^{\mathrm{sym}}=Y^{\mathrm{DIS}}+\ln \frac{k}{k^{\prime}}$ in (50) yields

$$
\begin{aligned}
G\left(k, k^{\prime}, Y^{\mathrm{sym}}\right. & \left.=Y^{\mathrm{DIS}}+\ln \frac{k}{k^{\prime}}\right) \equiv \tilde{G}\left(k, k^{\prime}, Y^{\mathrm{DIS}}\right) \\
& =\int_{-\infty}^{\infty} \frac{d \nu}{2 \pi^{2}} e^{\left[\bar{\alpha}_{\mu} \chi_{0}(\nu)+\bar{\alpha}_{\mu}^{2} \chi_{1}(\nu)\right]\left(Y^{\mathrm{DIS}}+\ln \frac{k}{k^{\prime}}\right)} H_{\frac{1}{2}+i \nu}(k)\left[H_{\frac{1}{2}+i \nu}\left(k^{\prime}\right)\right]^{*} \\
& \simeq \int_{-\infty}^{\infty} \frac{d \nu}{2 \pi^{2}} e^{\left[\bar{\alpha}_{\mu} \chi_{0}(\nu)+\bar{\alpha}_{\mu}^{2} \chi_{1}(\nu)\right] Y^{\mathrm{DIS}}} H_{\frac{1}{2}+i \nu}(k)\left[H_{\frac{1}{2}+i \nu}\left(k^{\prime}\right)\right]^{*}\left(1+\bar{\alpha}_{\mu} \chi_{0}(\nu) \ln \frac{k}{k^{\prime}}\right)
\end{aligned}
$$

where we have now put the rapidity-independent term into the prefactor. The NLO BFKL evolution equation in $Y$ DIS for the Green function $\tilde{G}$ has the kernel [24]

$$
K^{\mathrm{LO}+\mathrm{NLO}}(k, q)-\int d^{2} q^{\prime} K^{\mathrm{LO}}\left(k, q^{\prime}\right) \ln \frac{q^{\prime}}{k} K^{\mathrm{LO}}\left(q^{\prime}, q\right)
$$

In general we need to find the eigenfunctions and eigenvalues of this new kernel. However, it appears easier to simply try to cast Eq. (67) in the form where all the $k$ and $k^{\prime}$-dependence is contained in the product of a new eigenfunction and its complex conjugate (with momentum $k^{\prime}$ ). Since the new $k, k^{\prime}$-dependent factor in the parenthesis of the last line of Eq. (67) is real and can not be absorbed into the eigenfunctions, it has to be reabsorbed back into the exponent. (Alternatively we can note that $H_{\frac{1}{2}+i \nu}(k)$ are, in fact, eigenfunctions of the kernel (68).) To this end, with the NLO precision, we re-write $\ln \frac{k}{k^{\prime}}$ as a $\nu$-derivative acting on $\left(\frac{k^{2}}{k^{\prime 2}}\right)^{i \nu}$, perform a partial integration and include the term proportional to $Y^{\text {DIS }}$ back into the exponential, obtaining

$$
\tilde{G}\left(k, k^{\prime}, Y^{\mathrm{DIS}}\right)=\int_{-\infty}^{\infty} \frac{d \nu}{2 \pi^{2}} e^{\left[\bar{\alpha}_{\mu} \chi_{0}(\nu)+\bar{\alpha}_{\mu}^{2}\left(\chi_{1}(\nu)+\frac{i}{2} \chi_{0}(\nu) \chi_{0}^{\prime}(\nu)\right)\right] Y^{\mathrm{DIS}}} H_{\frac{1}{2}+i \nu}(k)\left[H_{\frac{1}{2}+i \nu}\left(k^{\prime}\right)\right]^{*}\left(1+\frac{i}{2} \bar{\alpha}_{\mu} \chi_{0}^{\prime}(\nu)\right) .
$$


It is clear from (69) that when this Green function $\tilde{G}$ is either acted upon by the kernel (68), or simply differentiated with respect to $Y^{\text {DIS }}$, the new eigenvalues

$$
\Delta^{\mathrm{DIS}}(\nu)=\bar{\alpha}_{\mu} \chi_{0}(\nu)+\bar{\alpha}_{\mu}^{2}\left(\chi_{1}(\nu)+\frac{i}{2} \chi_{0}(\nu) \chi_{0}^{\prime}(\nu)\right)
$$

obtained this way turn out to be shifted compared to (49) by $\bar{\alpha}_{\mu}^{2} \frac{i}{2} \chi_{0}(\nu) \chi_{0}^{\prime}(\nu)=-\bar{\alpha}_{\mu}^{2} \frac{1}{2} \chi_{0}(\gamma) \chi_{0}^{\prime}(\gamma)$, that is, by exactly the same term that was obtained in 24]. Note that we obtained this result without solving a new eigenvalue problem. In other words, the $H_{\gamma}(k)$ functions are also eigenfunctions for the non-symmetric NLO BFKL kernel of the DIS case. Notice also that the eigenvalues are not anymore symmetric under $\nu \rightarrow-\nu$ because now (69) is a solution of an evolution equation with a non-symmetric kernel.

Expanding $\Delta^{\mathrm{DIS}}(\gamma)$ in the powers of $\gamma$ around $\gamma=0$ and solving $\omega=\Delta^{\mathrm{DIS}}(\gamma)$ equation for $\gamma(\omega)$ one recovers the NNLO DGLAP anomalous dimension in the small- $x_{B}$ limit, as was demonstrated in [24].

\section{CONCLUSIONS AND OUTLOOK}

In this paper we have derived the solution for the NLO BFKL equation (50) by constructing its eigenfunctions using a perturbative expansion around the LO eigenfunctions. This expansion procedure can be used to construct solutions of the higher-order BFKL equation, as suggested in Eq. (52) above, providing a way of organizing the QCD perturbation series in the high energy scattering processes. It can already be applied to study the NNLO BFKL equation [51]. Our solution method can also be applied to the case of BFKL evolution with non-trivial azimuthal angle dependence, and to the non-forward BFKL equation as well.

Other immediate applications of our result include a construction of the NLO high-energy forward scattering amplitude for the $\gamma^{*} \gamma^{*}$ scattering process. The high-energy (Regge limit) scattering amplitude of $\gamma^{*} \gamma^{*}$ process, which encodes the hadronic contribution to, for example, the high-energy $e^{+} e^{-}$scattering, can be factorized into a convolution of the virtual photons impact factors and the BFKL ladder (Green function) exchange. Since the photon impact factors are known at NLO [64, 65], one can now use our NLO BFKL Green function (50) to fully construct the $\gamma^{*} \gamma^{*}$ forward scattering amplitude at NLO. This is also left for future work [51].

\section{Acknowledgments}

The authors are grateful to Ian Balitsky, Dima Kharzeev, and Al Mueller for helpful discussions. This research is sponsored in part by the U.S. Department of Energy under Grant No. DE-SC0004286.

\section{Appendix A: Orthogonality}

We want to show that the functions $H_{\frac{1}{2}+i \nu}(k)$ from Eq. (36) satisfy the orthogonality relation in Eq. (39). The relation is clearly satisfied by the LO BFKL kernel eigenfunctions. Substituting the eigenfunctions from Eq. (36) into Eq. (39) we obtain at order- $\bar{\alpha}_{\mu}$

$$
\begin{array}{r}
\int_{-\infty}^{\infty} d \nu f(\nu) \int \frac{d^{2} k}{k^{2}} k^{2 i\left(\nu-\nu^{\prime}\right)}\left\{\frac{i \beta_{2}}{2}\left[\frac{\chi_{0}(\nu)}{\chi_{0}^{\prime}(\nu)}-\frac{\chi_{0}\left(\nu^{\prime}\right)}{\chi_{0}^{\prime}\left(\nu^{\prime}\right)}\right] \ln ^{2} \frac{k^{2}}{\mu^{2}}+\frac{\beta_{2}}{2}\left[\frac{\partial}{\partial \nu}\left(\frac{\chi_{0}(\nu)}{\chi_{0}^{\prime}(\nu)}\right)+\frac{\partial}{\partial \nu^{\prime}}\left(\frac{\chi_{0}\left(\nu^{\prime}\right)}{\chi_{0}^{\prime}\left(\nu^{\prime}\right)}\right)\right] \ln \frac{k^{2}}{\mu^{2}}\right. \\
\left.+i\left(\operatorname{Im}\left[c_{1}(\nu)\right]-\operatorname{Im}\left[c_{1}\left(\nu^{\prime}\right)\right]\right) \ln \frac{k^{2}}{\mu^{2}}+c_{0}(\nu)+c_{0}^{*}\left(\nu^{\prime}\right)\right\}=0
\end{array}
$$

where $f(\nu)$ is an arbitrary test function. In the first term in (A1) we rewrite one power of the logarithm using

$$
\left(\ln \frac{k^{2}}{\mu^{2}}\right) k^{2 i\left(\nu-\nu^{\prime}\right)}=\left(-i \frac{\partial}{\partial \nu}-\ln \mu^{2}\right) k^{2 i\left(\nu-\nu^{\prime}\right)}
$$


and integrate by parts over $\nu$ to get

$$
\begin{array}{r}
\int_{-\infty}^{\infty} d \nu \int \frac{d^{2} k}{k^{2}} k^{2 i\left(\nu-\nu^{\prime}\right)}\left\{-\frac{\beta_{2}}{2} \frac{\partial}{\partial \nu}\left(\frac{\chi_{0}(\nu)}{\chi_{0}^{\prime}(\nu)}\right) f(\nu) \ln \frac{k^{2}}{\mu^{2}}-\frac{\beta_{2}}{2}\left[\frac{\chi_{0}(\nu)}{\chi_{0}^{\prime}(\nu)}-\frac{\chi_{0}\left(\nu^{\prime}\right)}{\chi_{0}^{\prime}\left(\nu^{\prime}\right)}\right] f^{\prime}(\nu) \ln \frac{k^{2}}{\mu^{2}}\right. \\
-\frac{i \beta_{2}}{2}\left[\frac{\chi_{0}(\nu)}{\chi_{0}^{\prime}(\nu)}-\frac{\chi_{0}\left(\nu^{\prime}\right)}{\chi_{0}^{\prime}\left(\nu^{\prime}\right)}\right] f(\nu) \ln \frac{k^{2}}{\mu^{2}} \ln \mu^{2}+\frac{\beta_{2}}{2}\left[\frac{\partial}{\partial \nu}\left(\frac{\chi_{0}(\nu)}{\chi_{0}^{\prime}(\nu)}\right)+\frac{\partial}{\partial \nu^{\prime}}\left(\frac{\chi_{0}\left(\nu^{\prime}\right)}{\chi_{0}^{\prime}\left(\nu^{\prime}\right)}\right)\right] f(\nu) \ln \frac{k^{2}}{\mu^{2}} \\
\left.+i\left(\operatorname{Im}\left[c_{1}(\nu)\right]-\operatorname{Im}\left[c_{1}\left(\nu^{\prime}\right)\right]\right) f(\nu) \ln \frac{k^{2}}{\mu^{2}}+2 \operatorname{Re}\left[c_{0}(\nu)\right] f(\nu)\right\}=0 .
\end{array}
$$

Canceling the first term with the first part of the fourth term, and applying the substitution (A2) to all remaining logarithms of $k^{2}$ yields after a few more cancellations

$$
\begin{array}{r}
\int_{-\infty}^{\infty} d \nu \int \frac{d^{2} k}{k^{2}} k^{2 i\left(\nu-\nu^{\prime}\right)}\left\{-\frac{i \beta_{2}}{2}\left[\frac{\chi_{0}(\nu)}{\chi_{0}^{\prime}(\nu)}-\frac{\chi_{0}\left(\nu^{\prime}\right)}{\chi_{0}^{\prime}\left(\nu^{\prime}\right)}\right] f^{\prime \prime}(\nu)+\frac{\beta_{2}}{2}\left[\frac{\chi_{0}(\nu)}{\chi_{0}^{\prime}(\nu)}-\frac{\chi_{0}\left(\nu^{\prime}\right)}{\chi_{0}^{\prime}\left(\nu^{\prime}\right)}\right] f^{\prime}(\nu) \ln \mu^{2}\right. \\
\left.-\left(\operatorname{Im}\left[c_{1}(\nu)\right]-\operatorname{Im}\left[c_{1}\left(\nu^{\prime}\right)\right]\right) f^{\prime}(\nu)\right\}=0
\end{array}
$$

where we have also employed Eq. (35). Integrating over $k$ gives a delta-function $\delta\left(\nu-\nu^{\prime}\right)$, which makes the expression in the curly brackets vanish, thus showing that Eq. (A4) is an identity.

We have, therefore, shown that the functions $H_{\frac{1}{2}+i \nu}(k)$ from Eq. (36) satisfy the orthogonality relation in Eq. (39) up to order- $\bar{\alpha}_{\mu}$, which is the limit of precision of the NLO approximation.

\section{Appendix B: Verifying the NNLO BFKL Solution Ansatz}

The goal of this Appendix is to verify whether the ansatz (62) solves Eq. (66). (The initial condition (22) is trivially satisfied by the ansatz.) Start with the right-hand-side of the equation (66): substituting the ansatz (62), replacing

$$
\ln \frac{k k^{\prime}}{\mu^{2}} \rightarrow \ln \frac{k^{\prime 2}}{\mu^{2}}-\frac{i}{2} \frac{\partial}{\partial \nu}
$$

in the arguments of the running couplings in the exponent with the derivative acing only on $\left(k^{2} / k^{\prime 2}\right)^{i \nu}$, and employing Eq. (63) we get

$$
\begin{aligned}
& \int d^{2} q K^{\mathrm{LO}+\mathrm{NLO}+\mathrm{NNLO}}(k, q) G\left(q, k^{\prime}, Y\right)=\int_{-\infty}^{\infty} \frac{d \nu}{2 \pi^{2} k k^{\prime}} \\
& \times \exp \left\{\bar{\alpha}_{\mu} \chi_{0}(\nu) Y\left[1-\bar{\alpha}_{\mu} \beta_{2}\left(\ln \frac{k^{\prime 2}}{\mu^{2}}-\frac{i}{2} \frac{\partial}{\partial \nu}\right)+\bar{\alpha}_{\mu}^{2} \beta_{2}^{2}\left(\ln \frac{k^{\prime 2}}{\mu^{2}}-\frac{i}{2} \frac{\partial}{\partial \nu}\right)^{2}+\bar{\alpha}_{\mu}^{2} \beta_{3}\left(\ln \frac{k^{\prime 2}}{\mu^{2}}-\frac{i}{2} \frac{\partial}{\partial \nu}\right)\right]\right. \\
& \left.+\bar{\alpha}_{\mu}^{2} \chi_{1}(\nu) Y\left[1-2 \bar{\alpha}_{\mu} \beta_{2}\left(\ln \frac{k^{\prime 2}}{\mu^{2}}-\frac{i}{2} \frac{\partial}{\partial \nu}\right)\right]+\bar{\alpha}_{\mu}^{3} \chi_{2}(\nu) Y\right\} \\
& \times\left\{\bar{\alpha}_{\mu} \chi_{0}(\nu)\left[1-\bar{\alpha}_{\mu} \beta_{2} \ln \frac{k^{2}}{\mu^{2}}+\bar{\alpha}_{\mu}^{2} \beta_{2}^{2} \ln ^{2} \frac{k^{2}}{\mu^{2}}+\bar{\alpha}_{\mu}^{2} \beta_{3} \ln \frac{k^{2}}{\mu^{2}}\right]+\bar{\alpha}_{\mu}^{2}\left[\frac{i}{2} \beta_{2} \chi_{0}^{\prime}(\nu)+\chi_{1}(\nu)\right]\left[1-2 \bar{\alpha}_{\mu} \beta_{2} \ln \frac{k^{2}}{\mu^{2}}\right]\right. \\
& \left.+\bar{\alpha}_{\mu}^{3}\left[\chi_{2}(\nu)+i \delta_{2}(\nu)\right]\right\}\left(\frac{k^{2}}{k^{\prime 2}}\right)^{i \nu},
\end{aligned}
$$

where now the $\nu$-derivatives act on everything to the right of the exponential. After a considerable algebra, involving numerous differentiations and integrations by parts, and expanding the exponential in the powers of running-coupling terms (that is, the terms containing $\beta_{2}$ and $\beta_{3}$ ) while keeping terms up to order- $\bar{\alpha}_{\mu}^{3}$ (in the power-counting of (599)) 
in the expression, we obtain for the right-hand-side

$$
\begin{aligned}
& \int d^{2} q K^{\mathrm{LO}+\mathrm{NLO}+\mathrm{NNLO}}(k, q) G\left(q, k^{\prime}, Y\right)= \\
& \int_{-\infty}^{\infty} \frac{d \nu}{2 \pi^{2} k k^{\prime}}\left(\frac{k^{2}}{k^{\prime 2}}\right)^{i \nu} e^{\left[\bar{\alpha}_{\mu} \chi_{0}(\nu)+\bar{\alpha}_{\mu}^{2} \chi_{1}(\nu)+\bar{\alpha}_{\mu}^{3} \chi_{2}(\nu)\right] Y}\left\{\bar{\alpha}_{\mu} \chi_{0}(\nu)\left[1-\bar{\alpha}_{\mu} \beta_{2} \ln \frac{k k^{\prime}}{\mu^{2}}+\left(\bar{\alpha}_{\mu} \beta_{2}\right)^{2}\left(\ln ^{2} \frac{k k^{\prime}}{\mu^{2}}+\frac{\chi_{0}^{\prime \prime}(\nu)}{4 \chi_{0}(\nu)}\right)\right]\right. \\
& -\left[\bar{\alpha}_{\mu} \chi_{0}(\nu)\right]^{2} Y\left[\bar{\alpha}_{\mu} \beta_{2} \ln \frac{k k^{\prime}}{\mu^{2}}-2\left(\bar{\alpha}_{\mu} \beta_{2}\right)^{2}\left(\ln ^{2} \frac{k k^{\prime}}{\mu^{2}}-\frac{\chi_{0}^{\prime \prime}(\nu)}{4 \chi_{0}(\nu)}+\frac{\chi_{0}^{\prime}(\nu)^{2}}{8 \chi_{0}(\nu)^{2}}\right)\right]+\bar{\alpha}_{\mu}^{2} \chi_{1}(\nu)\left[1-2 \bar{\alpha}_{\mu} \beta_{2} \ln \frac{k k^{\prime}}{\mu^{2}}\right] \\
& +\frac{1}{2}\left[\bar{\alpha}_{\mu} \chi_{0}(\nu)\right]^{3} Y^{2}\left(\bar{\alpha}_{\mu} \beta_{2}\right)^{2}\left(\ln ^{2} \frac{k k^{\prime}}{\mu^{2}}-\frac{\chi_{0}^{\prime \prime}(\nu)}{4 \chi_{0}(\nu)}\right)+\bar{\alpha}_{\mu}^{3} \chi_{0}(\nu) \beta_{3}\left(1+\bar{\alpha}_{\mu} \chi_{0}(\nu) Y\right) \ln \frac{k k^{\prime}}{\mu^{2}} \\
& \left.-3 \bar{\alpha}_{\mu}^{3} \chi_{0}(\nu) \chi_{1}(\nu) Y \bar{\alpha}_{\mu} \beta_{2} \ln \frac{k k^{\prime}}{\mu^{2}}+\frac{i}{2} \chi_{0}^{\prime}(\nu) \bar{\alpha}_{\mu}^{3} \beta_{3}-i \chi_{1}^{\prime}(\nu) \bar{\alpha}_{\mu}^{3} \beta_{2}+\bar{\alpha}_{\mu}^{3}\left[\chi_{2}(\nu)+i \delta_{2}(\nu)\right]\right\} .
\end{aligned}
$$

After a somewhat lesser amount of calculation involving a similar expansion of the exponential in the runningcoupling terms we obtain for the left-hand-side of Eq. (66)

$$
\begin{aligned}
\partial_{Y} G\left(k, k^{\prime}, Y\right) & =\int_{-\infty}^{\infty} \frac{d \nu}{2 \pi^{2} k k^{\prime}}\left(\frac{k^{2}}{k^{\prime 2}}\right)^{i \nu} e^{\left[\bar{\alpha}_{\mu} \chi_{0}(\nu)+\bar{\alpha}_{\mu}^{2} \chi_{1}(\nu)+\bar{\alpha}_{\mu}^{3} \chi_{2}(\nu)\right] Y}\left\{\bar{\alpha}_{\mu} \chi_{0}(\nu)\left[1-\bar{\alpha}_{\mu} \beta_{2} \ln \frac{k k^{\prime}}{\mu^{2}}+\left(\bar{\alpha}_{\mu} \beta_{2}\right)^{2} \ln ^{2} \frac{k k^{\prime}}{\mu^{2}}\right]\right. \\
& -\left[\bar{\alpha}_{\mu} \chi_{0}(\nu)\right]^{2} Y\left[\bar{\alpha}_{\mu} \beta_{2} \ln \frac{k k^{\prime}}{\mu^{2}}-2\left(\bar{\alpha}_{\mu} \beta_{2}\right)^{2} \ln ^{2} \frac{k k^{\prime}}{\mu^{2}}\right]+\bar{\alpha}_{\mu}^{2} \chi_{1}(\nu)\left[1-2 \bar{\alpha}_{\mu} \beta_{2} \ln \frac{k k^{\prime}}{\mu^{2}}\right] \\
& +\frac{1}{2}\left[\bar{\alpha}_{\mu} \chi_{0}(\nu)\right]^{3} Y^{2}\left(\bar{\alpha}_{\mu} \beta_{2}\right)^{2} \ln ^{2} \frac{k k^{\prime}}{\mu^{2}}+\bar{\alpha}_{\mu}^{3} \chi_{0}(\nu) \beta_{3}\left(1+\bar{\alpha}_{\mu} \chi_{0}(\nu) Y\right) \ln \frac{k k^{\prime}}{\mu^{2}} \\
& \left.-3 \bar{\alpha}_{\mu}^{3} \chi_{0}(\nu) \chi_{1}(\nu) Y \bar{\alpha}_{\mu} \beta_{2} \ln \frac{k k^{\prime}}{\mu^{2}}+\bar{\alpha}_{\mu}^{3} \chi_{2}(\nu)\right\} .
\end{aligned}
$$

Comparing the left-hand-side (B4) with the right-hand-side (B3) we see a complete agreement to order- $\bar{\alpha}_{\mu}^{2}$ (in the power-counting of (59) ). This provides an independent cross-check that Eq. (60) is, in fact, a solution of the NLO BFKL equation.

However, at order- $\bar{\alpha}_{\mu}^{3}$ the expressions in Eqs. (B4) and (B3) are not equal to each other. In fact, the difference is non-zero and is equal to

$$
\begin{aligned}
\partial_{Y} G\left(k, k^{\prime}, Y\right)-\int d^{2} q & K^{\mathrm{LO}+\mathrm{NLO}+\mathrm{NNLO}}(k, q) G\left(q, k^{\prime}, Y\right)=-\int_{-\infty}^{\infty} \frac{d \nu}{2 \pi^{2} k k^{\prime}}\left(\frac{k^{2}}{k^{\prime 2}}\right)^{i \nu} e^{\left[\bar{\alpha}_{\mu} \chi_{0}(\nu)+\bar{\alpha}_{\mu}^{2} \chi_{1}(\nu)+\bar{\alpha}_{\mu}^{3} \chi_{2}(\nu)\right] Y} \\
\times & \left\{\bar{\alpha}_{\mu}^{3} \beta_{2}^{2}\left[\frac{\chi_{0}^{\prime \prime}(\nu)}{4}+\bar{\alpha}_{\mu} \chi_{0}(\nu) Y\left(-\frac{\chi_{0}^{\prime \prime}(\nu)}{2}+\frac{\chi_{0}^{\prime}(\nu)^{2}}{4 \chi_{0}(\nu)}\right)-\left[\bar{\alpha}_{\mu} \chi_{0}(\nu) Y\right]^{2} \frac{\chi_{0}^{\prime \prime}(\nu)}{8}\right]\right. \\
+ & \left.\frac{i}{2} \chi_{0}^{\prime}(\nu) \bar{\alpha}_{\mu}^{3} \beta_{3}-i \chi_{1}^{\prime}(\nu) \bar{\alpha}_{\mu}^{3} \beta_{2}+\bar{\alpha}_{\mu}^{3} i \delta_{2}(\nu)\right\} .
\end{aligned}
$$

Hence the ansatz (62) can not be a solution of the NNLO BFKL equation, independent of what $\delta_{2}(\nu)$ is equal to.

Using Eq. (B5) we can correct the ansatz (62). First we notice that requiring the imaginary terms on the right of Eq. (B5) to separately vanish fixes $\delta_{2}(\nu)$ to be given by

$$
\delta_{2}(\nu)=-\frac{1}{2} \chi_{0}^{\prime}(\nu) \beta_{3}+\chi_{1}^{\prime}(\nu) \beta_{2} .
$$

Note that a complete perturbative construction of NNLO eigenfunctions, together with hermiticity of the NNLO BFKL kernel, would uniquely fix $\delta_{2}(\nu)$ : without doing that we may only conjecture that $\delta_{2}(\nu)$ is given by the expression (B6).

Finally, for $\delta_{2}(\nu)$ from Eq. (B6), we can correct the ansatz (마) by a multiplicative factor in the integrand, obtained 
by adding to unity the integral over $Y$ of the real term in the curly brackets on the right-hand-side of Eq. (B5):

$$
\begin{aligned}
& G\left(k, k^{\prime}, Y\right)=\int_{-\infty}^{\infty} \frac{d \nu}{2 \pi^{2} k k^{\prime}} e^{\left[\bar{\alpha}_{s}\left(k k^{\prime}\right) \chi_{0}(\nu)+\bar{\alpha}_{s}^{2}\left(k k^{\prime}\right) \chi_{1}(\nu)+\bar{\alpha}_{s}^{3}\left(k k^{\prime}\right) \chi_{2}(\nu)\right] Y}\left(\frac{k^{2}}{k^{\prime 2}}\right)^{i \nu} \\
& \times\left\{1+\left(\bar{\alpha}_{\mu} \beta_{2}\right)^{2}\left[-\frac{1}{24}\left(\bar{\alpha}_{\mu} Y\right)^{3} \chi_{0}(\nu)^{2} \chi_{0}^{\prime \prime}(\nu)+\frac{1}{4}\left(\bar{\alpha}_{\mu} Y\right)^{2} \chi_{0}(\nu)\left(\frac{\chi_{0}^{\prime}(\nu)^{2}}{2 \chi_{0}(\nu)}-\chi_{0}^{\prime \prime}(\nu)\right)+\bar{\alpha}_{\mu} Y \frac{\chi_{0}^{\prime \prime}(\nu)}{4}\right]\right\} .
\end{aligned}
$$

Let us stress once more that to verify the ansatz of Eqs. (B7) and (B6), and find the true value of $\delta_{2}(\nu)$ one has to construct NNLO eigenfunctions perturbatively with the help of Eq. (63), similar to how NLO eigenfunctions were found above. This is beyond the scope of the current work and is left for the future [51].

[1] J. Jalilian-Marian and Y. V. Kovchegov, Saturation physics and deuteron gold collisions at RHIC, Prog. Part. Nucl. Phys. 56 (2006) 104-231, hep-ph/0505052.

[2] H. Weigert, Evolution at small $x_{b j}$ : The Color Glass Condensate, Prog. Part. Nucl. Phys. 55 (2005) 461-565, hep-ph/0501087.

[3] E. Iancu and R. Venugopalan, The color glass condensate and high energy scattering in QCD, hep-ph/0303204.

[4] F. Gelis, E. Iancu, J. Jalilian-Marian, and R. Venugopalan, The Color Glass Condensate, Ann.Rev.Nucl.Part.Sci. 60 (2010) 463-489, arXiv:1002.0333.

[5] Y. V. Kovchegov and E. Levin, Quantum Chromodynamics at High Energy. Cambridge University Press, 2012.

[6] I. Balitsky, High-energy QCD and Wilson lines, hep-ph/0101042.

[7] I. Balitsky, Operator expansion for high-energy scattering, Nucl. Phys. B463 (1996) 99-160, hep-ph/9509348.

[8] I. Balitsky, Factorization and high-energy effective action, Phys. Rev. D60 (1999) 014020, hep-ph/9812311].

[9] Y. V. Kovchegov, Small-x $F_{2}$ structure function of a nucleus including multiple pomeron exchanges, Phys. Rev. D60 (1999) 034008, hep-ph/9901281.

[10] Y. V. Kovchegov, Unitarization of the BFKL pomeron on a nucleus, Phys. Rev. D61 (2000) 074018, hep-ph/9905214.

[11] J. Jalilian-Marian, A. Kovner, and H. Weigert, The Wilson renormalization group for low x physics: Gluon evolution at finite parton density, Phys. Rev. D59 (1998) 014015, hep-ph/9709432.

[12] J. Jalilian-Marian, A. Kovner, A. Leonidov, and H. Weigert, The Wilson renormalization group for low $x$ physics: Towards the high density regime, Phys. Rev. D59 (1998) 014014, hep-ph/9706377.

[13] E. Iancu, A. Leonidov, and L. D. McLerran, The renormalization group equation for the color glass condensate, Phys. Lett. B510 (2001) 133-144.

[14] E. Iancu, A. Leonidov, and L. D. McLerran, Nonlinear gluon evolution in the color glass condensate. I, Nucl. Phys. A692 (2001) 583-645, hep-ph/0011241.

[15] E. A. Kuraev, L. N. Lipatov, and V. S. Fadin, The Pomeranchuk singlularity in non-Abelian gauge theories, Sov. Phys. JETP 45 (1977) 199-204.

[16] E. A. Kuraev, L. N. Lipatov, and V. S. Fadin, Multi - reggeon processes in the yang-mills theory, Sov. Phys. JETP 44 (1976) 443-450.

[17] I. Balitsky and L. Lipatov, The Pomeranchuk Singularity in Quantum Chromodynamics, Sov.J.Nucl.Phys. 28 (1978) 822-829.

[18] J. L. Albacete, N. Armesto, J. G. Milhano, P. Quiroga-Arias, and C. A. Salgado, AAMQS: A non-linear QCD analysis of new HERA data at small-x including heavy quarks, Eur. Phys. J. C71 (2011) 1705, arXiv:1012.4408.

[19] J. L. Albacete and A. Dumitru, A model for gluon production in heavy-ion collisions at the LHC with rcBK unintegrated gluon densities, arXiv:1011.5161.

[20] I. I. Balitsky, Quark Contribution to the Small-x Evolution of Color Dipole, Phys. Rev. D 75 (2007) 014001, hep-ph/0609105.

[21] E. Gardi, J. Kuokkanen, K. Rummukainen, and H. Weigert, Running coupling and power corrections in nonlinear evolution at the high-energy limit, Nucl. Phys. A784 (2007) 282-340, hep-ph/0609087.

[22] Y. Kovchegov and H. Weigert, Triumvirate of Running Couplings in Small-x Evolution, Nucl. Phys. A 784 (2007) 188-226, hep-ph/0609090.

[23] Y. V. Kovchegov and H. Weigert, Quark loop contribution to BFKL evolution: Running coupling and leading-N(f) NLO intercept, Nucl. Phys. A789 (2007) 260-284, hep-ph/0612071.

[24] V. S. Fadin and L. N. Lipatov, BFKL pomeron in the next-to-leading approximation, Phys. Lett. B429 (1998) 127-134, hep-ph/9802290.

[25] M. Ciafaloni and G. Camici, Energy scale(s) and next-to-leading BFKL equation, Phys. Lett. B430 (1998) 349-354, hep-ph/9803389.

[26] I. Balitsky and G. A. Chirilli, Next-to-leading order evolution of color dipoles, Phys. Rev. D77 (2008) 014019, arXiv:0710.4330.

[27] Y. V. Kovchegov and A. H. Mueller, Running coupling effects in BFKL evolution, Phys. Lett. B439 (1998) 428-436, hep-ph/9805208. 
[28] E. Levin, The BFKL high-energy asymptotics in the next-to-leading approximation, hep-ph/9806228

[29] N. Armesto, J. Bartels, and M. Braun, On the second order corrections to the hard pomeron and the running coupling, Phys.Lett. B442 (1998) 459-469, hep-ph/9808340.

[30] M. Ciafaloni, M. Taiuti, and A. H. Mueller, Diffusion corrections to the hard pomeron, Nucl.Phys. B616 (2001) 349-366, hep-ph/0107009.

[31] D. A. Ross, The effect of higher order corrections to the BFKL equation on the perturbative pomeron, Phys. Lett. B431 (1998) 161-165, hep-ph/9804332.

[32] J. R. Andersen and A. Sabio Vera, Solving the BFKL equation in the next-to-leading approximation, Phys.Lett. B567 (2003) 116-124, hep-ph/0305236.

[33] J. R. Andersen and A. Sabio Vera, The gluon Green's function in the BFKL approach at next-to-leading logarithmic accuracy, Nucl.Phys. B679 (2004) 345-362, hep-ph/0309331.

[34] G. P. Salam, A resummation of large sub-leading corrections at small x, JHEP 07 (1998) 019, hep-ph/9806482.

[35] M. Ciafaloni, D. Colferai, and G. P. Salam, Renormalization group improved small-x equation, Phys. Rev. D60 (1999) 114036, hep-ph/9905566.

[36] M. Ciafaloni, D. Colferai, G. Salam, and A. Stasto, Renormalization group improved small $x$ Green's function, Phys.Rev. D68 (2003) 114003, hep-ph/0307188.

[37] S. Bondarenko, Conformal intercept of BFKL pomeron with NLO running coupling constant corrections, arXiv:0808.3175.

[38] Y. L. Dokshitzer, Calculation of the Structure Functions for Deep Inelastic Scattering and $e^{+} e^{-}$Annihilation by Perturbation Theory in Quantum Chromodynamics, Sov. Phys. JETP 46 (1977) 641-653.

[39] V. N. Gribov and L. N. Lipatov, Deep inelastic e p scattering in perturbation theory, Sov. J. Nucl. Phys. 15 (1972) 438-450.

[40] G. Altarelli and G. Parisi, Asymptotic Freedom in Parton Language, Nucl. Phys. B126 (1977) 298.

[41] D. J. Gross and F. Wilczek, Ultraviolet Behavior of Non-Abelian Gauge Theories, Phys. Rev. Lett. 30 (1973) $1343-1346$.

[42] H. Georgi and H. D. Politzer, Electroproduction scaling in an asymptotically free theory of strong interactions, Phys.Rev. D9 (1974) 416-420.

[43] E. Floratos, D. Ross, and C. T. Sachrajda, Higher Order Effects in Asymptotically Free Gauge Theories: The Anomalous Dimensions of Wilson Operators, Nucl.Phys. B129 (1977) 66-88.

[44] A. Vogt, S. Moch, and J. Vermaseren, The Three-loop splitting functions in QCD: The Singlet case, Nucl.Phys. B691 (2004) 129-181, hep-ph/0404111.

[45] A. Kotikov and L. Lipatov, NLO corrections to the BFKL equation in QCD and in supersymmetric gauge theories, Nucl.Phys. B582 (2000) 19-43, hep-ph/0004008.

[46] I. Balitsky and G. A. Chirilli, Conformal kernel for NLO BFKL equation in N=4 SYM, Phys.Rev. D79 (2009) 031502, arXiv:0812.3416.

[47] I. Balitsky and G. A. Chirilli, NLO evolution of color dipoles in N=4 SYM, Nucl.Phys. B822 (2009) 45-87, arXiv:0903.5326.

[48] I. Balitsky and G. A. Chirilli, High-energy amplitudes in N=4 SYM in the next-to-leading order, Int. J. Mod. Phys. A25 (2010) 401-410, arXiv:0911.5192.

[49] L. Lipatov, The Bare Pomeron in Quantum Chromodynamics, Sov.Phys.JETP 63 (1986) 904-912.

[50] H. D. Politzer, Reliable Perturbative Results for Strong Interactions?, Phys. Rev. Lett. 30 (1973) 1346-1349.

[51] G. A. Chirilli and Y. V. Kovchegov, in preparation.

[52] D. Y. Ivanov and A. Papa, Electroproduction of two light vector mesons in the next-to-leading approximation, Nucl.Phys. B732 (2006) 183-199, hep-ph/0508162.

[53] D. Y. Ivanov and A. Papa, Electroproduction of two light vector mesons in next-to-leading BFKL: Study of systematic effects, Eur.Phys.J. C49 (2007) 947-955, hep-ph/0610042.

[54] F. Caporale, D. Y. Ivanov, and A. Papa, BFKL resummation effects in the $\gamma^{*} \gamma^{*}$ total hadronic cross section, Eur.Phys.J. C58 (2008) 1-7, arXiv:0807.3231.

[55] R. Enberg, B. Pire, L. Szymanowski, and S. Wallon, BFKL resummation effects in $\gamma^{*} \gamma^{*} \rightarrow \rho \rho$, Eur.Phys.J. C45 (2006) 759-769, hep-ph/0508134.

[56] M. A. Braun, Reggeized gluons with a running coupling constant, Phys. Lett. B348 (1995) 190-195, hep-ph/9408261.

[57] E. Levin, Renormalons at low x, Nucl. Phys. B453 (1995) 303-333, hep-ph/9412345.

[58] W. E. Caswell, Asymptotic Behavior of Nonabelian Gauge Theories to Two Loop Order, Phys.Rev.Lett. 33 (1974) 244.

[59] D. Jones, Two Loop Diagrams in Yang-Mills Theory, Nucl.Phys. B75 (1974) 531.

[60] E. Egorian and O. Tarasov, Two Loop Renormalization of the QCD in an Arbitrary Gauge, Teor.Mat.Fiz. 41 (1979) $26-32$.

[61] R. D. Ball and S. Forte, All order running coupling BFKL evolution from GLAP (and vice-versa), Nucl.Phys. B742 (2006) 158-175, hep-ph/0601049.

[62] S. Marzani, R. D. Ball, P. Falgari, and S. Forte, BFKL at next-to-next-to-leading order, Nucl.Phys. B783 (2007) 143-175, arXiv:0704.2404.

[63] J. Bjorken, Asymptotic Sum Rules at Infinite Momentum, Phys.Rev. 179 (1969) 1547-1553.

[64] I. Balitsky and G. A. Chirilli, Photon impact factor and $k_{T}$-factorization for DIS in the next-to-leading order, Phys.Rev. D87 (2013) 014013, arXiv:1207.3844.

[65] I. Balitsky and G. A. Chirilli, Photon impact factor in the next-to-leading order, Phys.Rev. D83 (2011) 031502, arXiv:1009.4729. 